\title{
A Person-Centered Approach in Investigating a Mindfulness-Based Program for Adolescents with Autism Spectrum Disorder
}

\author{
Anna Ridderinkhof ${ }^{1} \cdot$ Mette Elmose $^{2}$ (I) Esther I. de Bruin ${ }^{1,3} \cdot$ René Blom $^{4} \cdot$ Sandra Salem-Guirgis ${ }^{5,6}$. \\ Jonathan A. Weiss ${ }^{5}$. Paulien van der Meer ${ }^{7}$. Nirbhay N. Singh ${ }^{8}$. Susan M. Bögels $s^{1,9}$
}

Accepted: 5 June 2021 / Published online: 23 August 2021

(c) The Author(s) 2021

\begin{abstract}
Objectives Adolescents with autism often experience comorbid internalizing disorders such as anxiety disorders or depression but the available evidence-based treatments to support the mental health of adolescents with autism are limited. The aim of this study was to investigate if and how MYmind, a mindfulness-based program (MBP) for youth with autism, could benefit adolescents with comorbid internalizing disorder(s).

Methods A person-centered approach with a multiple baseline design was used to investigate the effects of MYmind. Five adolescents with autism and an internalizing disorder took part in the 9-week MYmind program. The adolescents and their parents completed a daily questionnaire on their personal goals during a baseline phase, the intervention, a 2-month follow-up phase, and a 1-year follow-up phase. We analyzed the effects on their personal goals using visual inspection and statistical analysis for single-case designs. Also, we investigated potential processes of change by analyzing how changes were related over time.

Results Findings indicated that most, but not all, adolescents benefitted from the MBP. Four out of five adolescents showed medium-sized improvement in some of their personal goals. However, one adolescent reported a deterioration during the intervention and 2-month follow-up phase. Decreased worry preceded behavioral improvements in two adolescents, whereas other potential mechanisms of change showed inconclusive results.

Conclusion The findings indicated that most of the adolescents with autism and a comorbid internalizing disorder partially benefitted from the MBP.
\end{abstract}

Keywords Autism $\cdot$ Mindfulness-based program $\cdot$ Single-case design $\cdot$ Person-centered approach $\cdot$ Mechanisms of change

Mette Elmose

melandersen@health.sdu.dk

1 Research Institute of Child Development and Education, University of Amsterdam, Nieuwe Achtergracht 127, 1018 WS Amsterdam, The Netherlands

2 Department of Psychology, University of Southern Denmark, Campusvej 55, 5330 Odense M, Denmark

3 UvA Minds Academic Treatment Center, Banstraat 29, 1071 JW Amsterdam, The Netherlands

4 Child and Adolescent Psychiatry Center Karakter, Dr. Eeftinck Schattenkerkweg 1, 8025 BW Zwolle, The Netherlands

5 Department of Psychology, York University, 230 Behavioural Science Bldg, 4700 Keele Street, Toronto, ON M3J 1P3, Canada
6 Present Address: Schools of Health \& Wellness, George Brown College, Dockside Drive 416-415-5000 x6347, Toronto, Canada

7 Child and Adolescent Psychiatry Center De Bascule/Levvel, Rijksstraatweg 145, 1115AP Duivendrecht, Postbus : 303 / 1115 ZG, Duivendrecht, The Netherlands

8 Department of Psychiatry and Health Behavior, Medical College of Georgia, Augusta University, 997 St Sebastian Way, Augusta, GA 30912, USA

9 Department of Developmental Psychology, University of Amsterdam, Nieuwe Achtergracht 129-B, 1018 WS Amsterdam, The Netherlands 
Autism is a neurodevelopmental disability characterized by difficulties in social interaction and communication, and restricted, repetitive patterns of behavior or interest, including hyper- or hypo-reactivity to sensory stimuli (American Psychiatric Association (APA), 2013). The prevalence of comorbid internalizing conditions such as anxiety and mood disorders is high in people with autism (Ghaziuddin et al., 2002; Leyfer et al., 2006; Simonoff et al., 2008; Van Steensel et al., 2013a, b). Children with autism show a strong physiological reaction to stressors (Corbett et al., 2010; Spratt et al., 2012) and experience heightened stress and difficulties dealing with stress (Browning et al., 2009). Heightened stress responses have been associated with increased internalizing symptoms (e.g., Guerry \& Hastings, 2011; Kuhlman et al., 2014). Transitional phases such as adolescence are particularly difficult (Schall \& McDonough, 2010). Adolescents with autism experience more worries and stress with regard to leaving secondary education, as well as poorer abilities to deal with this stress, as compared to adolescents without autism (Browning et al., 2009). Nearly $40 \%$ of children with autism seem to have at least one comorbid anxiety disorder (Van Steensel et al., 2011) and around 13\% have a comorbid mood disorder (Van Steensel et al., 2013a, b). Children with autism and comorbid conditions have a significantly lower quality of life than children with autism without clinically elevated comorbid symptoms (Chiang \& Wineman, 2014). Compared to typically developing children, societal costs are 27 times higher for children with autism and comorbid anxiety disorders (Van Steensel et al., 2013a, b). Unfortunately, the availability of evidence-based treatments to support the mental health of adolescents with autism is limited (Newschaffer et al., 2007; Schall \& McDonough, 2010).

Mindfulness-based programs (MBPs) have been shown to decrease psychological and physiological stress in both healthy and clinical populations (e.g., Chiesa and Serretti, 2009; Morton et al., 2020; Pascoe et al., 2017) and to improve the mental health and wellbeing of children and adolescents (Dunning et al., 2019). The effects of MBPs have been found to be superior to active controls (Dunning et al., 2019) and largely equivalent to other evidence-based treatments (Farb et al., 2018; Goldberg et al., 2018; Kuyken et al., 2015). The interest in the application of MBPs also includes an interest in the potential effect for children and adolescents with autism and their families (e.g., De Bruin et al., 2015; Hwang et al., 2015; Ridderinkhof et al., 2018; Salem-Guirgis et al., 2019; Singh et al., 2011a, b). Children and adolescents with autism may benefit from an MBP by addressing their heightened stress and comorbid internalizing symptoms and disorders.

Mindfulness has been defined as attention to outward and inward experiences in the present moment, and approaching these experiences with an open, curious, and accepting attitude (Bishop et al., 2004). This quality is practiced in MBPs through meditation. Participants cultivate awareness and understanding of their thoughts, emotions, and bodily sensations, and practice viewing these experiences with a less judgmental and more accepting and compassionate attitude (Bishop et al., 2004; Segal et al., 2012). Instead of automatically reacting to and identifying with (negative) thoughts and emotions, participants learn to decenter. Decentering can be defined as the process of stepping outside their immediate train of thoughts and viewing these experiences from a distance as passing events in a wider field of awareness. In this shift in perspective, participants are able to recognize their thoughts as mental events rather than unchangeable truths and cultivate an allowing and even welcoming attitude (Segal et al., 2012). This decentering seems to be an important skill by which participants in MBPs learn to regulate distressing thoughts and emotions (Farb et al., 2018). Thus, in MBPs, self-regulatory coping strategies are learned (Kabat-Zinn, 1982). The cultivation of such self-regulatory strategies in MBPs might help adolescents with autism to reduce their stress and internalizing symptoms. MBPs may also help reduce problems with social interaction and communication. MBPs cultivate awareness and understanding of emotional processes, which could lead to an improved understanding of other people's emotions that improves children's social understanding and interaction. Also, increased present-moment awareness entails increased awareness of social interactions in that present moment as well; thus, interpersonal functioning might be improved by training mindfulness (Block-Lerner et al., 2007). These qualities make MBPs a potentially relevant treatment approach for children and adolescents with autism. However, the current evidence for the effect of MBPs for the autism population is still very limited compared to the more general evidence for MBPs.

The effects of an MBP on comorbid internalizing symptoms in autism have been investigated in studies of adults, consistently showing a significant decrease in depression, anxiety, and rumination (Kiep et al., 2015; Spek et al., 2013). Internalizing symptoms have also been investigated in studies of children and adolescents. In a pilot study of a 9-week MBP for 23 adolescents with autism and their parents, a reduction in adolescents' rumination and an increase in adolescents' quality of life were found up to 2 months after the MBP, although worry did not decrease (De Bruin et al., 2015). Another pilot study, investigating the effects of an MBP for six mothers of children with autism who in turn trained their children, found reductions in children's anxiety and thought problems (Hwang et al., 2015). A repeated measures study of a 9-week MBP for 45 children with autism and their parents found a reduction in children's internalizing symptoms and rumination up to 1 year after the MBP, and a reduction in stress 2 months after the MBP (Ridderinkhof et al., 2018). Finally, another repeated measures 
study of a 9-week MBP, which included a baseline period, for 23 adolescents found no significant change in internalizing symptoms, but a significant improvement in rumination, and aspects of emotion regulation (Salem-Guirgis et al., 2019).

The aforementioned studies all used a group format and combined an MBP for children with a mindful parenting program. Considering that the effects of a mindful parenting program alone could result in reduced internalizing and behavioral problems of the children (Hwang et al., 2015; Meppelink et al., 2016; Neece, 2014), it is yet unknown whether the reported benefits for children could be attributed to training the children, the parents, or both.

Research on MBPs for adolescents with autism is in its beginning stages and a study using a single-case experimental design could add to the evidence base. Single-case designs enable the researcher to study context-dependent knowledge that is lost if information is averaged in group designs. Context-dependent knowledge and experience are necessary to develop expertise in a field (Flyvbjerg, 2006). This knowledge provides information for future controlled studies such as RCTs (Borckardt et al., 2008; Drotar, 2009) and could help bridge the gap between clinical practice and science. Single-case designs also enable investigation of smaller populations for which large-group designs are less feasible. In addition, it allows researchers to individualize the outcomes on which treatment effects are evaluated, which is especially important in the autism population as their concerns are heterogeneous. Finally, by investigating the day-to-day differences in symptoms, the single-case design could provide understanding of how change unfolds over time during an MBP for adolescents with autism in a way that pre-post-follow-up group designs cannot (Borckardt et al., 2008).

In the current study, we used a multiple baseline design with five adolescents to investigate an MBP for adolescents with autism and comorbid internalizing disorders. The MBP was MYmind, a 9-week MBP for children and adolescents with autism and their parents (De Bruin et al., 2015), in individual sessions and without the parallel sessions of mindful parenting training. The format with individual sessions was chosen as it was expected that group sessions could hinder participation for some individuals with a high level of internalizing difficulties. We used a person-centered approach in which effects on the adolescents' personal mental health goals were viewed as the primary objective. We hypothesized that these personal goals would be improved by the MYmind program. As a secondary objective, we investigated how improvements in these personal goals were related over time with hypothesized process of change variables, including time spent on mindfulness practice, presentmoment attention, friendliness for self and others, worry, rumination, stress, and sleep.

\section{Method}

\section{Participants}

Participants were recruited at five different sites: three child mental health care centers in the Netherlands, namely UvA minds, Karakter, and De Bascule, the Institute of Psychology at the University of Southern Denmark in Denmark, and the Department of Psychology at the York University in Canada. Inclusion criteria were (1) an age of 14-21 years; (2) a DSM diagnosis of autism, further substantiated by the Autism Diagnostic Observation Schedule (ADOS; Lord et al., 2000, 2012); (3) clinically elevated internalizing symptoms as verified by meeting the criteria for at least one internalizing diagnosis on a semi-structured diagnostic interview (Anxiety Disorders Interview Schedule for children [ADIS-C/P], Silverman \& Albano, 1996; Structured Clinical Interview for DSM-5 Disorders for Children [SCID-Junior], Braet et al., 2016), and at least a borderline clinical score on the broadband scale internalizing problems of the Child Behavior Checklist (CBCL) and Youth Self Report (YSR; Achenbach \& Rescorla, 2001); and (4) an IQ of 80 or higher as indicated by at least two subtests of a Wechsler Scale of Intelligence, namely Matrix reasoning and Vocabulary, or Block design and Vocabulary (e.g., Wechsler, 2011). Exclusion criteria were (1) inadequate mastery of the language of the intervention; (2) diagnosed with oppositional defiant disorder (ODD) or conduct disorder (CD); (3) presence of current suicidal risk according to the clinician; (4) diagnosed with current non-treated psychotic disorder or posttraumatic stress disorder (PTSD); (5) receiving another ongoing psychological intervention (apart from "stable" medication); and (6) living away from family home. The families decided themselves who would be the participating parent. Written informed consent was obtained from all participants.

Five adolescents with autism, all with internalizing concerns, participated. Names are pseudonyms. Mark, a 15-year-old boy with autism, met the classification criteria for autism spectrum on the ADOS, and for generalized anxiety disorder, illness anxiety disorder, and depressive disorder on the SCID-junior. Previously, he received social skills and group counseling support. He lived at home with his parents and attended high school. His mother completed the parent-reports and participated in one mindfulness session. Mark's personal goals were to decrease his negative thoughts and to increase his positive thoughts.

Thomas, a 17-year-old boy with autism, met the classification criteria for autism spectrum on the ADOS, and for generalized anxiety disorder, social anxiety disorder, and persistent depressive disorder on the ADIS. He was 
taking aripiprazole and fluoxetine for his depression. Previously, he underwent eye-movement desensitization and reprocessing therapy (EMDR), cognitive behavior therapy (CBT), and psychomotor therapy. He lived at home with his parents, dropped out of special education at high school, and went to a work-experience facility. His father participated in the parent-reports and one mindfulness session. Thomas' personal goals were to increase his focus at work, to become aware when he was thinking negatively, and to decrease being seized by negative thinking.

David, a 19-year-old boy with autism, scored two points below the cutoff for autism spectrum on the ADOS, and met the classification criteria for generalized anxiety disorder and social anxiety disorder on the ADIS. Previously, he received CBT. He lived at home with his mother and attended an autism class in high school. Based on current and prior challenges David's mother was compensated economically enabling her to support David's school attendance and general everyday functioning. His mother participated in the parent-reports and one mindfulness session. David's personal goals were to decrease feeling anxious and to feel more relaxed in his body.

Steven, a 14-year-old boy with autism, met the classification criteria for autism spectrum on the ADOS, and for social anxiety disorder on the ADIS. He had not received any treatment before taking part in the MYmind program. He lived at home with his parents and attended middle school. His father participated in the parent-reports and one mindfulness session. Steven's personal goals were to increase feeling confident and to decrease feeling worried.

Linda, an 18-year-old girl with autism, met the classification criteria for autism spectrum on the ADOS, and for generalized anxiety disorder, specific phobia, and depressive disorder in remission on the ADIS. She was taking citalopram for her depressive symptoms. Previously, she received social skills training, inpatient mental health care, family therapy, psychotherapy, and other antidepressant medication. She lived with her parents, attended vocational education, and did volunteer work. Her father participated in the parentreports and one mindfulness session. Linda's personal goals were to fall asleep more easily, to be less irritated by sounds, and to be less irritated by her mother.

\section{Procedure}

A multiple baseline design (Barlow et al., 2009) across participants was used, consisting of four phases: baseline, intervention, 2-month follow-up, and 1-year follow-up. A pre-intervention appointment took place with the adolescent, one parent, the mindfulness trainer, and the research assistant at each location. During this appointment, the intervention and the study procedures were explained, and relevant background information was obtained. Also, the adolescent's personal goals for the intervention were discussed. The adolescents and parents who agreed to participate in the study were asked to complete a daily questionnaire during the baseline, intervention, 2-month follow-up, and 1-year follow-up phases via the online application Qualtrics. The research assistant monitored the questionnaires and if necessary reminded participants about the daily questionnaire by a phone call or sending a text, to help increase compliance.

The length of the baseline varied irregularly among the participants in accordance with the multiple baseline design (Barlow et al., 2009). Baseline length, intended to be of a minimum of 7 days and a maximum of 28 days, was assigned to the participants in the order of inclusion. Due to practicalities the length of the baseline varied between 5 and 79 days (Table 1). The intervention phase lasted as long as it took to complete the nine sessions of the MYmind program. This varied among participants because of personal circumstances such as holidays or illness in between. The 2-month follow-up phase lasted 9 weeks and was conducted between the final intervention session and the booster session. The 1-year follow-up phase lasted 2 weeks and was conducted 1 year after the final intervention session. Ethical approval was received for each country. In the Netherlands, the Medical Ethics Committee of the Amsterdam Medical Center approved the study. In Canada, the Human Participants Ethics Review Sub-Committee of York University approved the study. In Denmark,

Table 1 Participant characteristics and descriptives of the time-series

\begin{tabular}{lllllllllr}
\hline Client & TIQ & CBCL INT & YSR INT & $\begin{array}{l}\text { Baseline } \\
\text { \#days }\end{array}$ & $\begin{array}{l}\text { Intervention } \\
\text { \# days }\end{array}$ & $\begin{array}{l}\text { 2-month f-u } \\
\text { \# days }\end{array}$ & $\begin{array}{l}\text { 1-year f-u } \\
\text { \# days }\end{array}$ & $\begin{array}{l}\% \text { missing } \\
\text { self-report }\end{array}$ & $\begin{array}{l}\% \text { missing } \\
\text { parent-report }\end{array}$ \\
\hline Mark & 100 & 70 & 69 & 5 & 64 & 58 & 14 & 19.1 & 24.1 \\
Thomas & 109 & 76 & 67 & 7 & 67 & 66 & 14 & 45.5 & 36.4 \\
David & 112 & 83 & 79 & 11 & 94 & 67 & 14 & 3.2 & 2.2 \\
Steven & 113 & 66 & 67 & 18 & 58 & 48 & 14 & 15.9 & 2.9 \\
Linda & 119 & 74 & 61 & 79 & 41 & 62 & 14 & 25.0 & 25.0 \\
\hline
\end{tabular}

Note. $T I Q$, total intelligence quotient estimate; $C B C L I N T$, Child Behavior Checklist Internalizing Scale T-score at baseline; YSR INT, Youth Self Report Internalizing Scale T-score at baseline; $f$ - $u$, follow-up. \% missing is based on the number of days missing a report relative to the total number of days for all phases 
the Regional Committees on Health Research Ethics for Southern Denmark approved the study.

Intervention The MYmind program is a manual based MBP including nine weekly sessions of $1.5 \mathrm{~h}$ and an additional booster session 9 weeks later (De Bruin et al., 2015; Ridderinkhof et al., 2018) with group-based sessions for youth and parallel mindful parenting sessions for parents. In the present study, the MYmind protocol was presented to adolescents individually and parents did not participate. Each session included psychoeducation about the session theme, mindfulness meditation practices, informal practices to apply during daily living, inquiry about practices, and discussions about home practices. The nine session themes were as follows: (1) what is attention?, (2) attention for the body, (3) attention for the breath, (4) attention for stress, (5) attention for emotions, (6) attention for external distractors such as sounds, (7) attention for internal distractors such as thoughts and feelings of self and others, (8) attention for changes, and (9) attention for mindfulness after the training. Mindfulness practices included breathing meditation, 3-min breathing space, body scan meditation, seeing meditation, hearing meditation, thought meditation, walking meditation, yoga, and informal mindfulness exercises (Kabat-Zinn, 1982; Segal et al., 2012). The mindfulness practices were theorized to enable the adolescents to cultivate focused attention, awareness of inward and outward experiences, self-control, and an accepting stance toward experiences. The program was tailored to the specific needs of youth with autism, such as the session on dealing with unexpected changes, a more explicit structure, less verbal guidance during the meditations, a more structured inquiry with closed questions, and more explanation on the goal and purpose of meditation practices (see De Bruin et al., 2015 and Ridderinkhof et al., 2018 for more details on the MYmind program).

The parent that completed the assessments also received one session of mindfulness training of $1.5 \mathrm{~h}$ at the beginning of the intervention phase so that they were aware of the content of the intervention their adolescent were to receive. This session included explanation about the MYmind program, psychoeducation on mindfulness and autism, a discussion on how they could support their child with the home practices without being obtrusive, and several mindfulness practices such as the breathing meditation. In addition, the ninth session of the adolescent program and the booster session was extended by $30 \mathrm{~min}$ in which the parent joined in an evaluation of the intervention and discussion on how to proceed with mindfulness practices in the upcoming period in which no weekly session took place. During the booster session, a report of the outcomes of the daily questionnaire was also discussed with the adolescents and their parents.
The five instructors were therapists experienced in working with adolescents with autism and were trained in MYmind for youth with ADHD/autism and their parents. They had all instructed at least one MYmind training for youth with autism before this study. They also had a personal practice of mindfulness meditation. The research assistant at each location was present during the sessions and took part in all practices and inquiries as a participant-observer, to function as a role model and to enhance the discussion during inquiry.

Materials The daily questionnaire included items on the adolescents' personal goals and on potential process of change variables. The personal goals were designed in collaboration with the adolescents and the parent and reviewed with two members of the research team (AR and EdB). For each participant, two or three personal goals of high priority that were related to their distress and internalizing symptoms were recorded using The Goal Attainment Scale (GAS; Cardillo \& Choate, 1994). One goal was positively worded (e.g., "I want to feel more bodily relaxed") and one was negatively worded (e.g., "I want to have fewer negative thoughts"). For each personal goal, a daily assessable item was formulated on the internal state and on its reflection in the adolescent's behavior as described by the adolescent and parent, such as "Today, I had negative thoughts" and "Today, I withdrew from activities." Attention was paid throughout the process to use the phrasing of the adolescents.

The potential process of change variables included items on mindfulness practice time in minutes, present-moment attention, being friendly for self, being friendly for others, worry, rumination, stress, and sleep. Present-moment attention represents an aspect of mindfulness, and friendliness for self and others represents aspects of self-compassion, which were noted as mechanisms of change of MBPs in other populations (Kuyken et al., 2010). Worry, rumination, stress, and sleep problems were selected as internalizing symptoms of adolescents with autism which could hamper their daily functioning and seem to be decreased by MBPs (Chiesa \& Serretti, 2009; Winbush et al., 2007). The rumination item was for example formulated as "Today, I thought again and again about my feelings and/or problems," and the stress item was formulated as "Today, I felt stressed." Parents' daily questionnaire included the items on their child's behavioral reflection of the personal goals, and items on their child's present-moment attention, stress, being friendly for self, and being friendly for others. For example, "Today, my child made decisions immediately" and "Today, my child was present in the common rooms when at home." All items were rated on a 7-point scale ranging from 1 (not at all) to 7 (all the time). 


\section{Data Analyses}

Data from the daily questionnaire were inspected visually (Lane \& Gast, 2014) using means, standard deviations, ranges of the variables for each phase, and the graphs. We interpreted changes as a decrease or increase if the difference in means between phases or the graph showed a downward or upward trend. In addition, we used Simulation Modeling Analysis (SMA; Borckardt et al., 2008) with 5000 simulations to statistically analyze the data for each individual. SMA is a bootstrapping methodology that accounts for autocorrelation and can handle short baseline phases. We conducted a level change and a slope change analysis. The level change analysis tested whether the mean level of one phase differed from the mean level of the adjacent phase. Pearson's $r$ correlation coefficients were produced as indicators of effect size. For interpreting the magnitude of the effect size, we followed the suggestions of Cohen (1992) that the effect size be considered small, medium, and large, when Pearson's $r$ varies around $0.1,0.3$ and 0.5 respectively. The slope change analysis tested the relation of the observed data with an a priori defined slope model. We hypothesized the slope to be stable during the baseline phase and to increase (or decrease for negatively formulated items) during the intervention phase (slope vector 2 in SMA), to further increase (or decrease) during the 2-month follow-up phase (slope vector 4 in SMA), and to be stable during the 1-year follow-up phase (slope vector 3 in SMA). Pearson's $r$ correlation indicated the degree of concordance with the a priori defined slope vector. Both level change and slope change were analyzed on the same data, and therefore, a Bonferroni adjustment of the alpha level was made $(\alpha=0.05 / 2=0.025$; Cabin \& Mitchell, 2000).

Furthermore, we investigated the relation between the personal goals and the self-reported process of change variables over time using cross-lagged correlations. With crosslagged correlations, the data were analyzed pairwise to test whether one variable led or lagged the other. We tested -5 to +5 adjacent lags and corrected the alpha level for the number of lags (Borckardt et al., 2008). These analyses were only conducted for the process of change variables that improved during the intervention phase with $p<0.05$, and for the personal goals that improved significantly during the intervention or 2-month follow-up phase.

SMA and cross-correlations cannot handle missing data, but missing data are inevitable when participants are asked to report daily. Therefore, missing data were imputed using the expectation maximization procedure (Dempster et al., 1977), which seems acceptable for time-series when autocorrelation is below 0.80 (Smith et al., 2012). Little's (1988) MCAR test was conducted to test whether the data were missing completely at random for each adolescent separately using both the self-reported and parent-reported daily data of all phases. These tests were nonsignificant for Mark, Thomas, David, and Linda ( $p=0.220-0.624)$, thus missing data were then imputed. For Steven's data, this test was significant if all daily data were used $(p<0.000)$. This is probably due to the fact that if 1 day was missed, all items on that day were missed. We re-analyzed the data with the variables we used for the SMA analyses for this case, namely the personal goal items and the stress items. Little's MCAR test was nonsignificant ( $p=0.171)$; thus, missing data on these variables were then imputed.

\section{Results}

Results of the personal goals and the change analyses are presented and discussed for each adolescent. All means and standard deviations of the daily items for each adolescent per phase can be seen in Table 2 .

\section{Mark}

For Mark's goal 1, negative thinking, visual inspection (Fig. 1) combined with SMA (Table 3) showed a mediumsized statistically significant decrease during the intervention phase as compared to the baseline phase, continuing further during the 2-month follow-up, and during the 1-year follow-up. The reflecting behavior, withdrawing to his room, decreased statistically significant with a large and a medium effect size according to self-report, but not according to parent-report (Table 3). For Mark's goal 2, positive thinking, visual inspection combined with SMA showed a nonsignificant decrease during the intervention as compared to the baseline but a statistically significant increase during the 2-month follow-up and during the 1-year follow-up of a medium and large effect size respectively (Table 2 ). The reflecting behavior, being open, increased statistically significant with a medium effect size according to self-report, but not according to parent-report (Table 3). Thus, the goals to decrease negative thinking and to increase positive thinking improved according to Mark, but not according to his parent.

The process of change variables that improved during the intervention as compared to the baseline were worry, stress, friendly for self, and friendly for others $(p<0.043)$. The cross-lagged correlations (Table 4 ) indicated that during the intervention decreased worry preceded decreased withdrawn behavior (Fig. 2). Also, decreased stress preceded increased open behavior. Increased being friendly for self followed decreased negative thoughts and withdrawn behavior, and also preceded decreased withdrawn behavior during the intervention. Increased being friendly for others preceded decreased withdrawn behavior during the intervention but followed this decrease during the 2-month follow-up (Table 5). This indicated that decreased worry and stress 


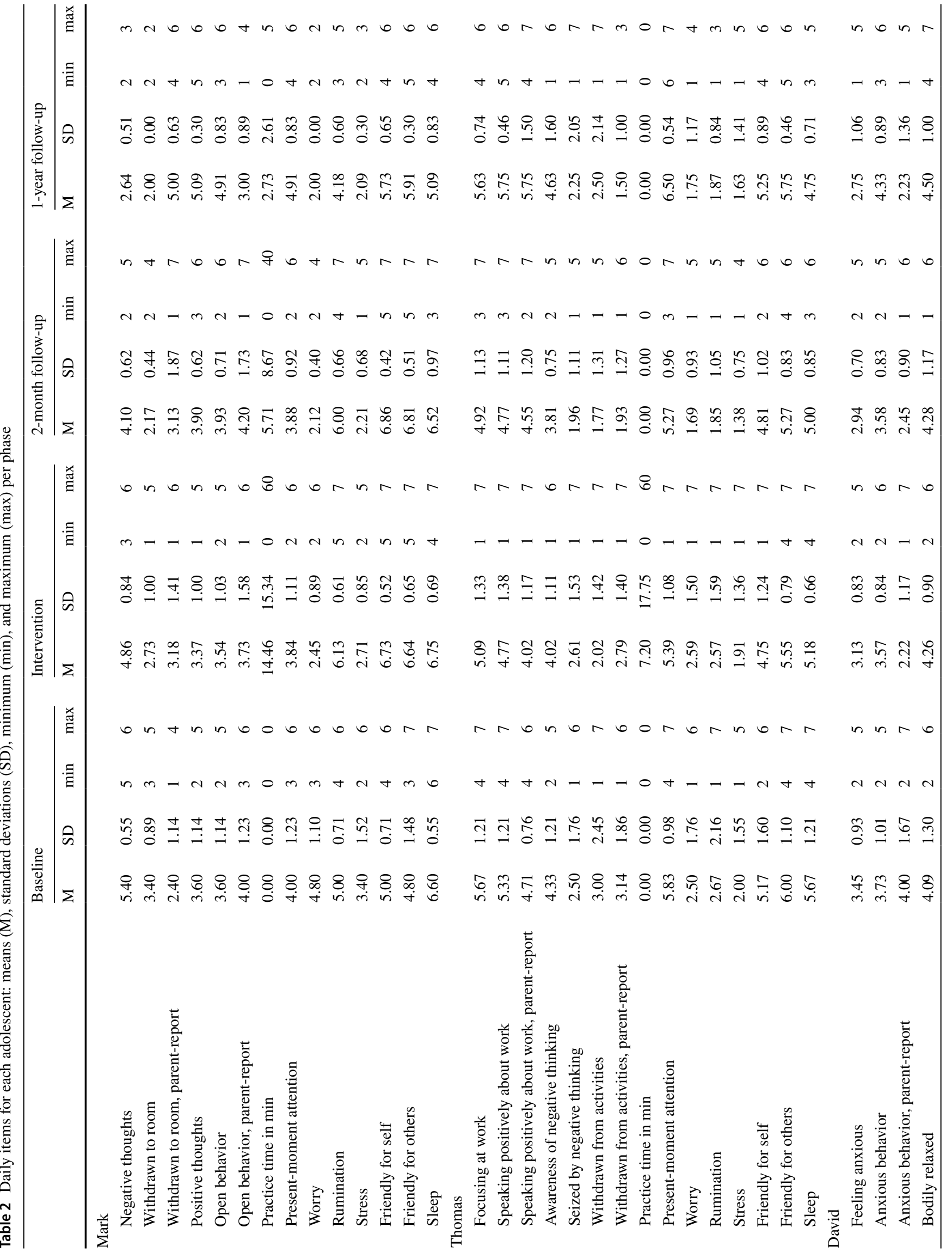




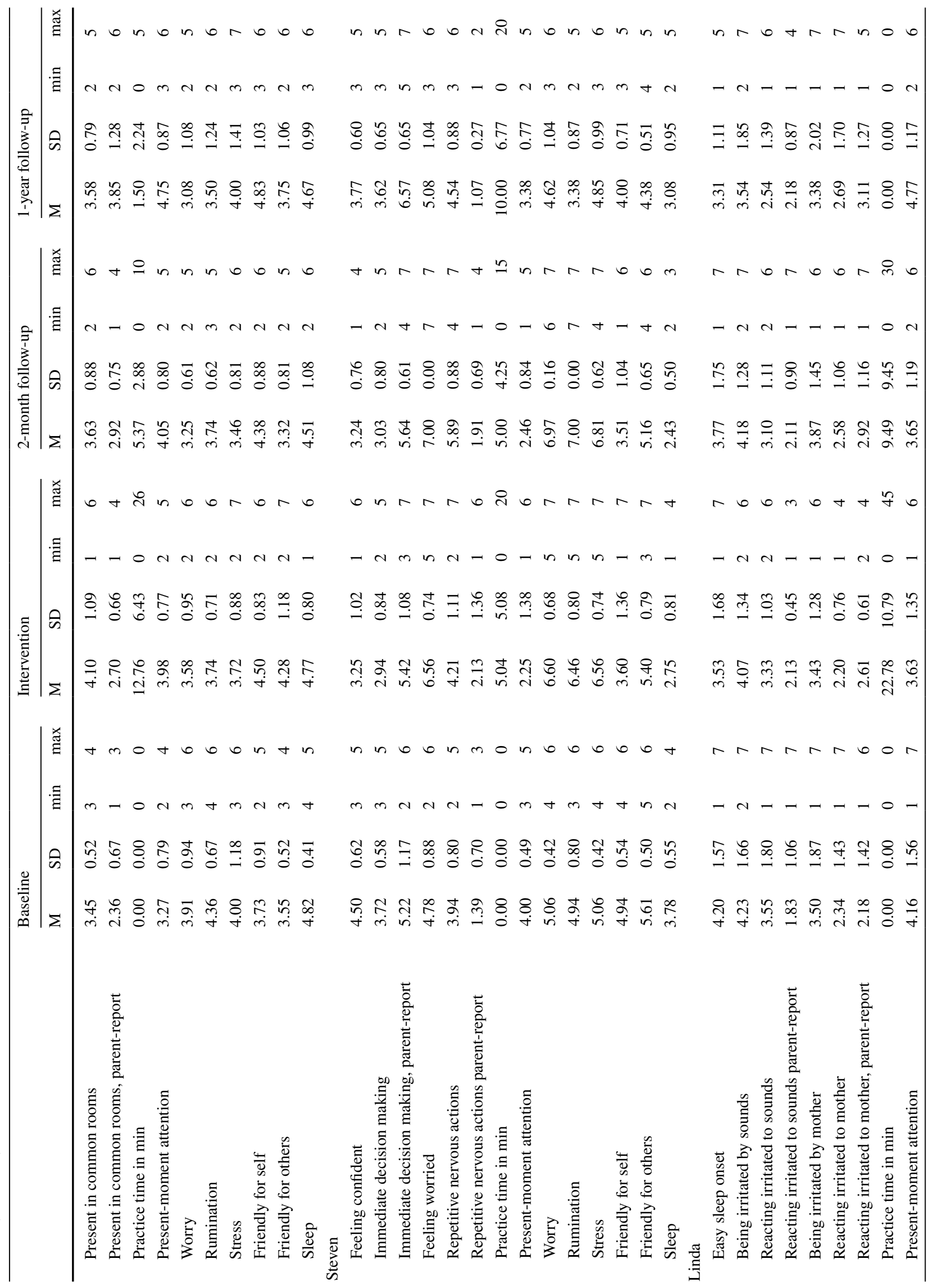




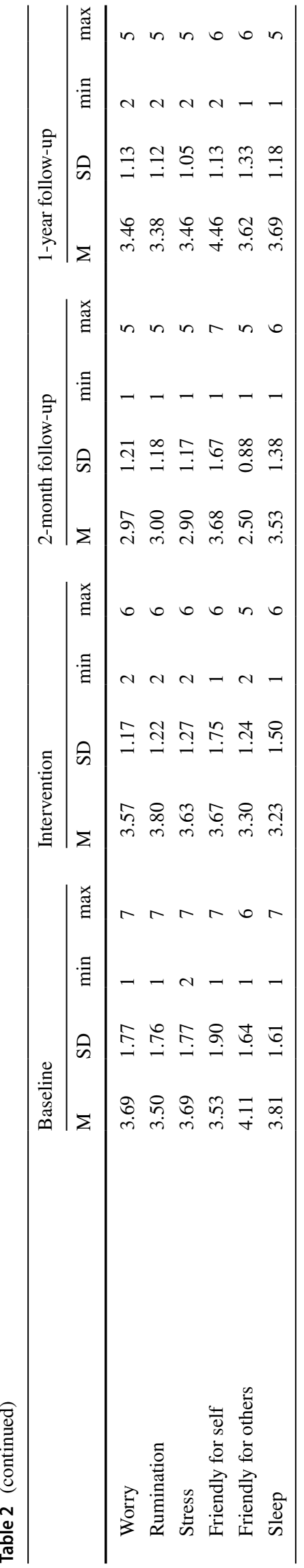

preceded the behavioral improvements in Mark's personal goals, whereas increased being friendly both preceded and followed the improvements in his personal goals.

\section{Thomas}

For Thomas' goal 1, focus at work, visual inspection indicated that this, opposite to expectation, decreased during the intervention phase compared to the baseline, further decreased during the 2-month follow-up, but increased during the 1-year follow-up. These changes were not statistically significant (Table 3 ). The reflecting behavior, speaking positively about work, only significantly improved with a medium effect size from the 2-month to the 1-year follow-up according to self-report, but not according to parent-report (Table 3). For goal 2, awareness of negative thinking, a similar pattern was seen with no significant level or slope changes (Table 3). No behavioral reflection was formulated for this goal. Visual inspection indicated that being seized by negative thinking, goal 3 , remained at the same level during the baseline and the intervention, decreased during the 2-month follow-up, and remained stable during the 1-year follow-up (Fig. 1). The decrease during the 2-month follow-up as compared to the intervention phase was statistically significant and medium sized (Table 3 ). The reflecting behavior, withdrawing from activities, did not change according to self-report but decreased significantly with a medium effect size according to parent-report. Thus, the goal to be less seized by negative thinking seemed improved during the 2-month follow-up according to Thomas and his parent. The other two goals did not show improvement. No process of change variables significantly improved during the intervention phase for Thomas (Table 3). Therefore, no cross-lagged correlation analyses were conducted.

\section{David}

Visual inspection indicated that David's goal 1, feeling anxious, decreased during the intervention as compared to the baseline, further decreased during the 2-month follow-up and during the 1-year follow-up. This downward trend was not statistically significant (Table 3 ). The reflecting anxious behavior, including withdrawing and isolating himself, significantly decreased with a medium effect size according to parent-report, but not according to self-report (Table 3). Visual inspection indicated that David's goal 2, feeling bodily relaxed, increased during the intervention as compared to the baseline, increased during the 2-month follow-up, and stabilized during the 1 -year follow-up. Only the slope change from 2-month to 1 -year follow-up was statistically significant. The reflecting behavior, being present in the common rooms when at home, significantly increased with a medium effect size 

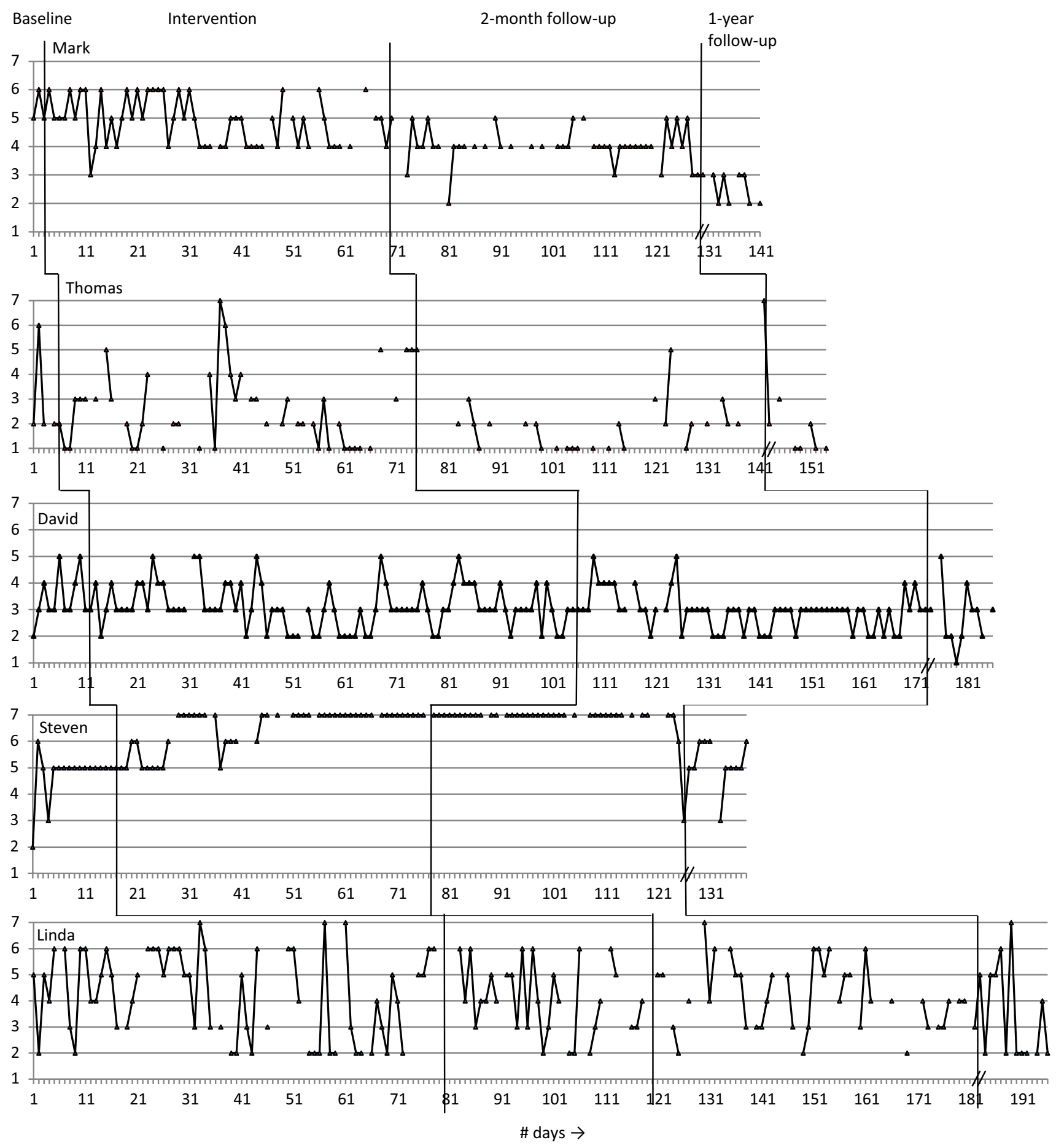

Fig. 1 Ratings of one personal goal for each client during baseline, intervention, 2-month follow-up, and 1-year follow-up. The negatively formulated goals are presented, so a decline represents an

according to parent-report, but not according to self-report (Table 3). Thus, the goal to feel less anxious improved, but only statistically significant according to parent-report, and the goal to feel more bodily relaxed improved according to David and his parent. improvement. Mark: negative thoughts; Thomas: seized by negative thinking; David: feeling anxious; Steven: feeling worried; Linda: irritated by sounds

The process of change variables mindfulness practice time, present-moment attention, worry, and friendly for self improved during the intervention as compared to the baseline $(p<0.045)$. The cross-lagged correlations (Table 5) indicated that increased mindfulness practice time preceded 
Table 3 Simulation modeling analysis results on the personal goal items

\begin{tabular}{|c|c|c|c|c|c|c|}
\hline & \multicolumn{2}{|c|}{$\begin{array}{l}\text { Baseline to inter- } \\
\text { vention }\end{array}$} & \multicolumn{2}{|c|}{$\begin{array}{l}\text { Intervention to } \\
2 \text {-month follow- } \\
\text { up }\end{array}$} & \multicolumn{2}{|c|}{$\begin{array}{l}\text { 2-month to } \\
\text { 1-year follow-up }\end{array}$} \\
\hline & Level & Slope & Level & Slope & Level & Slope \\
\hline \multicolumn{7}{|l|}{ Mark } \\
\hline Negative thoughts & -0.20 & $-0.34 *$ & $-0.41^{*}$ & $-0.44^{*}$ & $-0.62 *$ & $-0.36^{*}$ \\
\hline Withdrawn to room & -0.20 & $-0.53 *$ & -0.25 & $-0.38 *$ & -0.23 & -0.10 \\
\hline Withdrawn to room parent-report & 0.16 & 0.12 & -0.01 & 0.02 & $0.39 *$ & 0.29 \\
\hline Positive thoughts & -0.04 & 0.17 & $0.28 *$ & $0.30 *$ & $0.56^{*}$ & $0.32 *$ \\
\hline Open behavior & 0.00 & 0.26 & 0.21 & $0.27 *$ & $0.41 *$ & 0.22 \\
\hline Open behavior parent-report & -0.04 & 0.13 & 0.10 & 0.15 & $-0.29 *$ & -0.10 \\
\hline Practice time in min & 0.25 & & $-0.30^{*}$ & & -0.16 & \\
\hline Present-moment attention & -0.04 & -0.08 & & & & \\
\hline Worry & $-0.59 *$ & $-0.60 *$ & & & & \\
\hline Rumination & $0.45^{*}$ & 0.21 & & & & \\
\hline Stress & -0.22 & $-0.40^{\wedge}$ & & & & \\
\hline Friendly for self & $0.66^{*}$ & $0.53 *$ & & & & \\
\hline Friendly for others & $0.58 *$ & $0.45^{\wedge}$ & & & & \\
\hline Sleep & 0.04 & -0.02 & & & & \\
\hline \multicolumn{7}{|l|}{ Thomas } \\
\hline Focusing on work & -0.14 & -0.09 & -0.02 & -0.04 & 0.18 & 0.06 \\
\hline Speaking positively about work & -0.13 & -0.12 & 0.08 & 0.03 & $0.27 *$ & 0.09 \\
\hline Speaking positively about work parent-report & -0.17 & -0.04 & 0.16 & 0.20 & 0.03 & 0.16 \\
\hline Awareness of negative thinking & -0.09 & 0.02 & -0.03 & 0.01 & 0.19 & 0.16 \\
\hline Seized by negative thinking & 0.01 & 0.01 & $-0.27 *$ & -0.25 & 0.07 & -0.03 \\
\hline Withdrawn from activities & -0.19 & -0.09 & -0.15 & -0.16 & 0.15 & 0.01 \\
\hline Withdrawn from activities parent-report & -0.12 & -0.14 & $-0.26^{*}$ & $-0.28 *$ & 0.05 & -0.07 \\
\hline Practice time in min & 0.11 & & $-0.22 *$ & & -0.06 & \\
\hline Present-moment attention & -0.13 & -0.11 & & & & \\
\hline Worry & 0.00 & -0.07 & & & & \\
\hline Rumination & -0.03 & -0.05 & & & & \\
\hline Stress & -0.02 & 0.06 & & & & \\
\hline Friendly for self & -0.11 & 0.12 & & & & \\
\hline Friendly for others & -0.21 & -0.23 & & & & \\
\hline Sleep & -0.19 & -0.15 & & & & \\
\hline \multicolumn{7}{|l|}{ David } \\
\hline Feeling anxious & -0.12 & -0.19 & -0.12 & -0.21 & -0.12 & -0.29 \\
\hline Anxious behavior & -0.05 & -0.09 & 0.01 & -0.05 & 0.30 & 0.04 \\
\hline Anxious behavior, parent-report & $-0.41 *$ & $-0.42 *$ & 0.11 & -0.06 & -0.06 & -0.25 \\
\hline Bodily relaxed & 0.05 & 0.20 & 0.01 & 0.18 & 0.10 & $0.44^{*}$ \\
\hline Present in common rooms & 0.18 & -0.01 & -0.23 & -0.19 & 0.01 & 0.23 \\
\hline Present in common rooms, parent-report & 0.16 & $0.31 *$ & 0.16 & $0.33 *$ & 0.33 & $0.52^{*}$ \\
\hline Practice time in min & $0.54 *$ & & $-0.56^{*}$ & & $-0.50 *$ & \\
\hline Present-moment attention & $0.27^{\wedge}$ & 0.19 & & & & \\
\hline Worry & -0.10 & $-0.29^{\wedge}$ & & & & \\
\hline Rumination & -0.26 & -0.25 & & & & \\
\hline Stress & -0.09 & -0.18 & & & & \\
\hline Friendly for self & $0.27 *$ & $0.25 *$ & & & & \\
\hline Friendly for others & 0.19 & 0.01 & & & & \\
\hline Sleep & -0.02 & 0.07 & & & & \\
\hline \multicolumn{7}{|l|}{ Steven } \\
\hline Feeling confident & $-0.49^{*}$ & $-0.54 *$ & -0.01 & -0.10 & 0.27 & 0.28 \\
\hline
\end{tabular}


Table 3 (continued)

\begin{tabular}{|c|c|c|c|c|c|c|}
\hline & \multicolumn{2}{|c|}{$\begin{array}{l}\text { Baseline to inter- } \\
\text { vention }\end{array}$} & \multicolumn{2}{|c|}{$\begin{array}{l}\text { Intervention to } \\
\text { 2-month follow- } \\
\text { up }\end{array}$} & \multicolumn{2}{|c|}{$\begin{array}{l}\text { 2-month to } \\
\text { 1-year follow-up }\end{array}$} \\
\hline & Level & Slope & Level & Slope & Level & Slope \\
\hline Immediate decision making & $-0.40 *$ & $-0.62 *$ & 0.04 & -0.10 & $0.32 *$ & $0.38^{*}$ \\
\hline Immediate decision making parent-report & 0.07 & 0.06 & -0.09 & 0.02 & $0.54 *$ & 0.24 \\
\hline Feeling worried & $0.68 *$ & $0.75^{*}$ & 0.32 & $0.51 *$ & $-0.79 *$ & -0.49 \\
\hline Repetitive nervous actions & 0.12 & 0.34 & $0.57 *$ & $0.62 *$ & $-0.45^{*}$ & -0.20 \\
\hline Repetitive nervous actions parent-report & 0.25 & 0.31 & -0.09 & 0.02 & $-0.51 *$ & -0.21 \\
\hline Practice time in min & $0.46^{*}$ & & 0.01 & & $0.41 *$ & \\
\hline \multicolumn{7}{|l|}{ Linda } \\
\hline Easy sleep onset & -0.17 & -0.08 & 0.04 & 0.17 & -0.13 & 0.13 \\
\hline Being irritated by sounds & -0.05 & -0.11 & 0.05 & -0.06 & -0.19 & -0.22 \\
\hline Reacting irritated to sounds & -0.07 & -0.11 & -0.09 & $-0.25^{*}$ & -0.21 & $-0.36^{*}$ \\
\hline Reacting irritated to sounds parent-report & 0.10 & 0.08 & 0.01 & -0.04 & -0.04 & -0.09 \\
\hline Being irritated by mother & 0.01 & 0.05 & 0.12 & 0.18 & -0.15 & 0.00 \\
\hline Reacting irritated to mother & -0.07 & -0.06 & 0.19 & 0.18 & 0.04 & 0.06 \\
\hline Reacting irritated to mother parent-report & 0.19 & 0.20 & 0.09 & 0.16 & -0.05 & 0.06 \\
\hline Practice time in min & $0.80 *$ & & $-0.49 *$ & & $-0.44 *$ & \\
\hline Present-moment attention & -0.15 & -0.10 & & & & \\
\hline Worry & -0.05 & -0.14 & & & & \\
\hline Rumination & 0.09 & 0.01 & & & & \\
\hline Stress & -0.04 & -0.15 & & & & \\
\hline Friendly for self & 0.05 & 0.08 & & & & \\
\hline Friendly for others & $-0.25^{\wedge}$ & -0.14 & & & & \\
\hline Sleep & -0.16 & -0.09 & & & & \\
\hline
\end{tabular}

Note. Results are presented as Pearson's $r$ correlation coefficients. Level change reflects the change in mean level between the phases. Slope change reflects the correlation with an a priori specified slope vector. For baseline to intervention, this was stable to increasing. For intervention to 2-month follow-up, this was increasing to increasing. For 2-month follow-up to 1-year follow-up, this was increasing to stable

${ }^{\wedge} p<0.05 ;{ }^{*} p<0.025$

increased parent-reported presence in the common rooms during the 2-month follow-up. Also, decreased worry preceded decreased parent-reported anxious behavior and increased parent-reported presence in the common rooms during the intervention and the 2-month follow-up. In addition, during the 2-month follow-up, increased presentmoment attention preceded decreased parent-reported anxious behavior. Increased parent-reported presence in the common rooms both preceded and followed increased present-moment attention during the 2-month follow-up (Fig. 3). This indicated that increased practice time and present-moment attention and decreased worry preceded the parent-reported behavioral improvements of David.

\section{Steven}

Visual inspection indicated that Steven's goal 1, feeling confident, opposite to expectation, decreased during the intervention phase as compared to the baseline, remained on this lower level during the 2-month follow-up, and slightly increased during the 1-year follow-up. This decrease in feeling confident was statistically significant with a large effect size, and the increase during the 1-year follow-up was not (Table 2). Opposite to expectation, the reflecting behavior, making decisions immediately, significantly decreased during the intervention as compared to the baseline with a medium and large effect size according to self-report. However, it significantly increased during the 1-year follow-up as compared to the 2-month follow-up with a medium effect size according to self-report and with a large effect size according to parent-report (Table 3). Visual inspection indicated that Steven's goal 2, feeling worried, also opposite to expectation, increased during the intervention phase compared to the baseline, and increased during the 2-month follow-up, but decreased during the 1-year followup compared to the 2-month follow-up. Both the increase and decrease in worry were statistically significant with large effect sizes (Table 3 ). The reflecting behavior, repetitive nervous actions, significantly increased during the 2-month follow-up compared to the intervention according to self-report with a large effect size, but significantly decreased with a large effect 


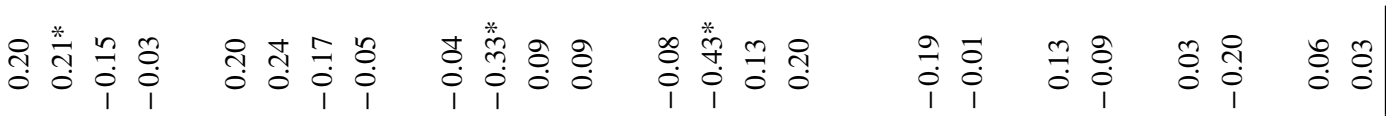

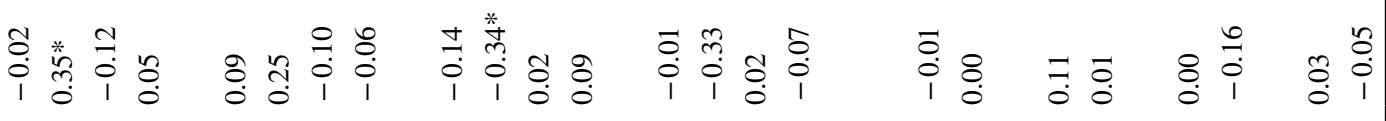

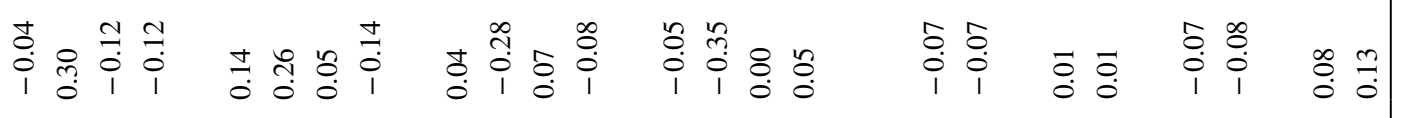

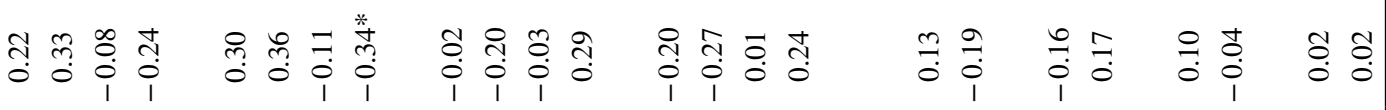

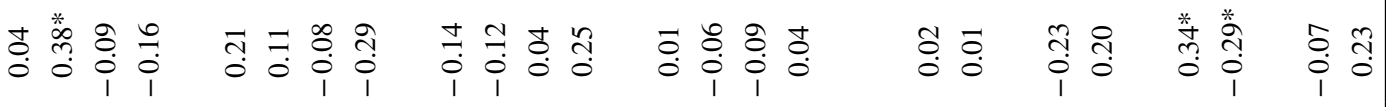

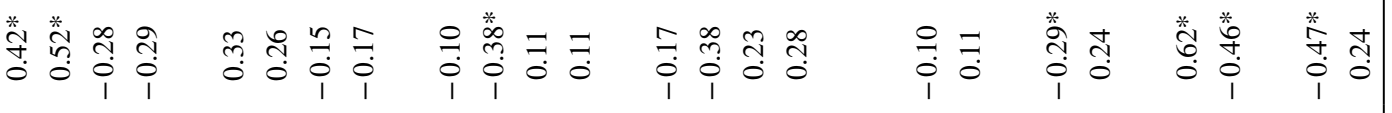

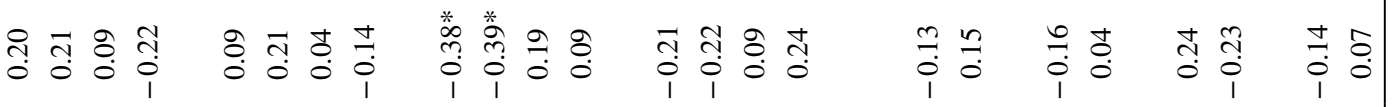

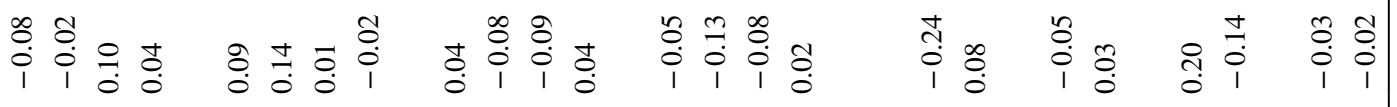

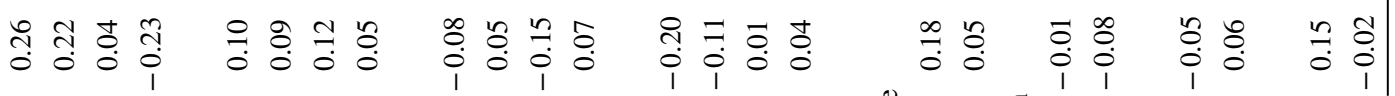

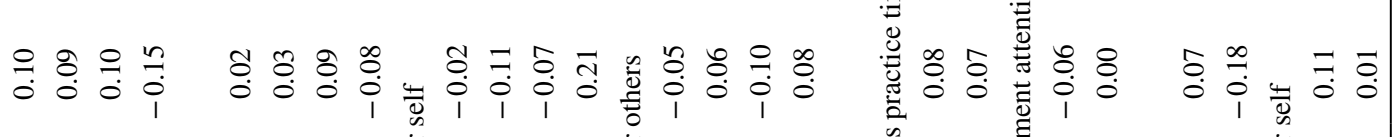

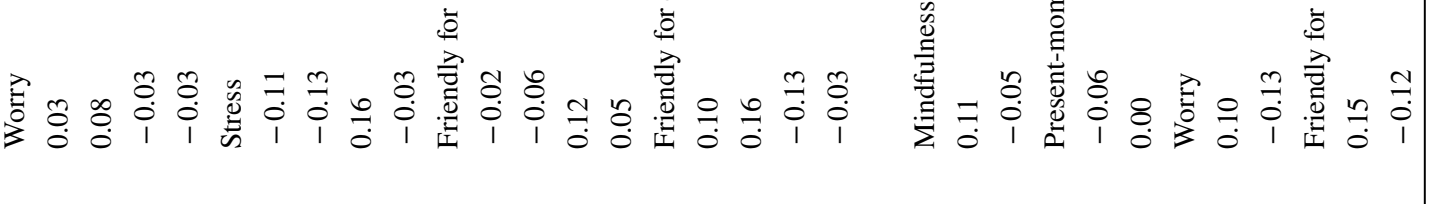


size from the 2-month follow-up into the 1-year follow-up according to self- and parent-report. Thus, the goals to feel more confident and less worried deteriorated during the intervention and the 2-month follow-up but improved during the 1-year follow-up. These improvements during the 1-year follow-up were above baseline levels according to parent-report, but not according to self-report. As Steven's personal goals did not improve during the intervention or the 2-month follow-up, no cross-lagged correlation analyses were conducted.

\section{Linda}

Visual inspection indicated that Linda's goal 1, easy sleep onset, fluctuated during and between all phases. It decreased during the intervention as compared to the baseline, increased during the 2-month follow-up, and decreased again during the 1-year follow-up. These changes were not statistically significant (Table 3). For this goal, no behavioral reflection was formulated. For Linda's goal 2, being irritated by sounds, visual inspection indicated fluctuation during all phases, decrease during the intervention as compared to the baseline, and further decrease during the 2-month and 1-year follow-up. The decrease was not statistically significant (Table 2). The reflecting behavior, reacting irritated to sounds, significantly decreased according to self-report with a medium effect size, but not according to parent-report (Table 3). Visual inspection indicated that Linda being irritated by her mother, goal 3, also fluctuated during all phases, and slightly decreased during the intervention as compared to the baseline, increased during the 2-month follow-up as compared to the intervention, and decreased again during the 1 -year follow-up. These changes were not statistically significant (Table 3). The reflecting behavior, reacting irritated to her mother, did not change according to both self- and parent-report (Table 3). Thus, the goal to be less irritated by sounds improved during the 2-month follow-up according to Linda's report of her behavior, but the goals to fall asleep more easily and to be less irritated by her mother did not improve.

The process of change variable mindfulness practice time improved during the intervention phase as compared to the baseline $(p<0.001)$. The cross-lagged correlations indicated no relation between mindfulness practice time and self-reported irritated reaction to sounds during the intervention and the 2-month follow-up.

\section{Discussion}

In this study, we used a person-centered approach to investigate the effects of the MYmind program for adolescents with autism and a comorbid internalizing disorder. The results showed variation across the five cases. 
Fig. 2 Cross-lagged correlation results between worry and withdrawn behaviour of Mark during the intervention. This indicated that the decrease in Mark's withdrawn behaviour was preceded by the decrease in worry

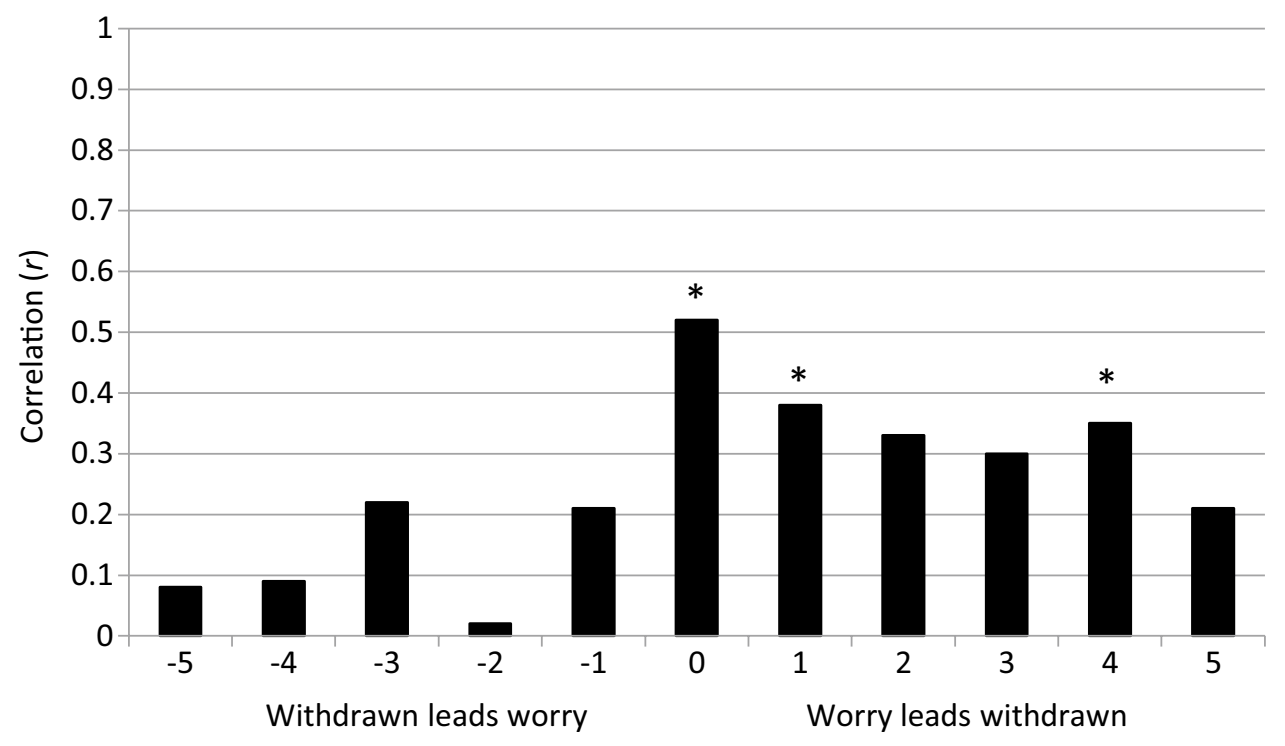

Mark reported improvement in both his personal goals, but this was not confirmed by his parent's reports. As reported by themselves or by their parent, Thomas and Linda improved on one out of three of their personal goals and David improved on both his goals but only during one of the three time periods. Steven reported deterioration on his personal goals, namely increased worried feeling and decreased feelings of confidence, during the intervention and 2-month follow-up. However, his goals did not deteriorate according to his parent and had improved at the 1-year follow-up. We also investigated how improvements on adolescents' personal goals related to process of change variables over time for Mark, David, and Linda. Results indicated that decreased worry preceded behavioral improvements in personal goals for both Mark and David. Improvement in personal goals was linked to decreased stress and increased being friendly for Mark only, and to increased mindfulness practice time and increased presentmoment attention for David only. David being friendly for self, and Linda's mindfulness practice time, was also investigated but no significant relation was found to personal goals. Process of change was not investigated for Thomas and Steve as no significant change was found for the change variables during the intervention period.

The findings of this study indicated that participating in the MYmind program coincided with a significant reduction in one or more of the identified internalizing problems for the adolescents, such as decreased negative thinking and withdrawal from activities. This extends the literature on MBPs for children and adolescents with autism showing decreased rumination, stress, and internalizing symptoms, as well as decreased social responsivity symptoms, externalizing symptoms, and attention problems (De Bruin et al., 2015; Hwang et al., 2015; Ridderinkhof et al., 2018;
Salem-Guirgis et al., 2019) and increased self-management of physical aggression (Singh et al., 2011a, b).

Although most adolescents showed improvement in part of their personal goals, Steven reported a deterioration in his personal goals during the MYmind intervention and Thomas, David, and Linda only partly improved on their personal goals (e.g., one goal, or one time period only) while putting considerable effort into following the program. This to some degree contrasts with the more positive improvement reported for MBPs in RCTs (Dunning et al., 2019; Goldberg et al., 2018). However, RCTs on MBPs may overly report positive results, which may result in overestimating the effects of MBPs in practice (Coronado-Montoya et al., 2016). Scholars call for monitoring and reporting possible negative effects of MBPs in study trials, in order to decrease exaggerations of the benefits and safety of MBPs (Van Dam et al., 2018; Wong et al., 2018). Negative effects of MBPs as well as of all kinds of psychosocial treatments are unfortunately an understudied phenomenon (e.g., Rozental et al., 2018; Van Dam et al., 2018) and only $25 \%$ of study trials investigating MBPs actively assesses adverse effects (Van Dam et al., 2018). Still, adverse effects in relation to meditation, such as symptoms of psychosis, depersonalization, anxiety, and panic, have been documented (Lindahl et al., 2017; Van Dam et al., 2018).

Steven's increase in worry and decrease in feelings of confidence could reflect adverse effects of the MYmind program for him. While we did not systematically assess adverse effects in the present study, we did monitor all cases closely. In the evaluation of the outcomes on the daily questionnaire with Steven and his parent, they shared that the MYmind program had been very beneficial for Steven. For Steven, the MYmind program started during his summer holidays and Steven started at a new school during the 
Table 5 Cross-lagged correlation results during the 2-month follow-up phase

\begin{tabular}{|c|c|c|c|c|c|c|c|c|c|c|c|}
\hline Lag & -5 & -4 & -3 & -2 & -1 & 0 & 1 & 2 & 3 & 4 & 5 \\
\hline & \multicolumn{11}{|c|}{ Process of change variable } \\
\hline \multicolumn{12}{|l|}{ Mark } \\
\hline & Worry & & & & & & & & & & \\
\hline Negative thoughts & -0.12 & 0.16 & -0.06 & 0.22 & -0.16 & $0.43 *$ & 0.00 & 0.15 & 0.15 & 0.06 & -0.07 \\
\hline Withdrawn to room & -0.06 & 0.10 & -0.08 & 0.18 & -0.20 & $0.51 *$ & -0.01 & 0.00 & 0.01 & 0.10 & -0.06 \\
\hline Positive thoughts & 0.02 & -0.12 & 0.11 & -0.03 & 0.12 & $-0.39^{*}$ & -0.03 & -0.18 & -0.18 & -0.09 & 0.04 \\
\hline \multirow[t]{2}{*}{ Open behavior } & 0.10 & 0.09 & 0.09 & 0.08 & -0.04 & -0.23 & 0.03 & 0.09 & -0.30 & -0.09 & 0.03 \\
\hline & Stress & & & & & & & & & & \\
\hline Negative thoughts & -0.20 & 0.03 & -0.12 & 0.17 & -0.22 & $0.36^{*}$ & 0.09 & 0.19 & 0.09 & 0.07 & -0.01 \\
\hline Withdrawn to room & -0.16 & 0.07 & 0.01 & 0.18 & -0.24 & $0.41 *$ & 0.14 & -0.15 & -0.08 & 0.10 & -0.07 \\
\hline Positive thoughts & 0.07 & -0.02 & 0.08 & -0.11 & 0.19 & $-0.35^{*}$ & -0.12 & -0.17 & -0.11 & -0.10 & -0.02 \\
\hline \multirow[t]{2}{*}{ Open behavior } & 0.12 & 0.29 & -0.02 & 0.02 & -0.07 & $-0.36^{*}$ & -0.11 & 0.07 & -0.06 & -0.10 & -0.02 \\
\hline & \multicolumn{11}{|c|}{ Friendly for self } \\
\hline Negative thoughts & 0.00 & -0.13 & -0.06 & -0.05 & 0.02 & 0.03 & 0.02 & -0.13 & 0.08 & -0.05 & 0.01 \\
\hline Withdrawn to room & 0.04 & -0.22 & -0.05 & 0.04 & -0.05 & -0.29 & 0.23 & 0.04 & -0.16 & -0.07 & 0.02 \\
\hline Positive thoughts & 0.04 & 0.10 & 0.03 & 0.17 & -0.05 & 0.01 & 0.02 & 0.02 & 0.10 & 0.16 & -0.12 \\
\hline \multirow[t]{2}{*}{ Open behavior } & -0.05 & 0.03 & 0.09 & 0.04 & 0.04 & -0.03 & -0.10 & -0.10 & 0.16 & -0.04 & -0.09 \\
\hline & \multicolumn{11}{|c|}{ Friendly for others } \\
\hline Negative thoughts & -0.11 & -0.09 & 0.14 & -0.13 & -0.01 & -0.23 & 0.05 & -0.18 & -0.01 & 0.06 & 0.17 \\
\hline Withdrawn to room & 0.07 & $-0.35 *$ & -0.07 & -0.11 & 0.19 & $-0.51^{*}$ & 0.14 & 0.20 & 0.09 & 0.03 & 0.17 \\
\hline Positive thoughts & 0.16 & 0.02 & -0.16 & 0.18 & 0.06 & 0.28 & 0.00 & 0.17 & 0.06 & 0.04 & -0.12 \\
\hline Open behavior & -0.10 & -0.09 & 0.01 & 0.03 & 0.13 & 0.11 & 0.01 & 0.01 & 0.17 & 0.00 & -0.09 \\
\hline \multicolumn{12}{|l|}{ David } \\
\hline & \multicolumn{11}{|c|}{ Mindfulness practice time } \\
\hline Anxious behavior, parent-report & 0.01 & -0.18 & -0.16 & -0.08 & -0.02 & -0.12 & -0.17 & -0.30 & -0.16 & -0.14 & -0.10 \\
\hline \multirow[t]{2}{*}{ Present in common rooms, parent-report } & 0.15 & 0.22 & 0.30 & 0.37 & 0.32 & 0.37 & $0.47 *$ & $0.44 *$ & 0.27 & 0.26 & 0.20 \\
\hline & \multicolumn{11}{|c|}{ Present-moment attention } \\
\hline Anxious behavior, parent-report & 0.00 & -0.08 & -0.27 & -0.13 & -0.14 & $-0.44^{*}$ & $-0.50 *$ & -0.25 & -0.13 & -0.20 & -0.23 \\
\hline \multirow[t]{2}{*}{ Present in common rooms, parent-report } & 0.09 & 0.14 & $0.37 *$ & $0.39 *$ & 0.37 & $0.50 *$ & $0.47 *$ & 0.34 & 0.24 & 0.37 & 0.18 \\
\hline & \multicolumn{11}{|l|}{ Worry } \\
\hline Anxious behavior, parent-report & -0.02 & 0.09 & 0.30 & 0.10 & 0.00 & $0.55^{*}$ & $0.50 *$ & 0.20 & 0.16 & 0.29 & 0.16 \\
\hline \multirow[t]{2}{*}{ Present in common rooms, parent-report } & -0.14 & -0.22 & -0.35 & -0.35 & -0.22 & $-0.55^{*}$ & $-0.48 *$ & -0.26 & -0.30 & -0.25 & -0.14 \\
\hline & \multicolumn{11}{|c|}{ Friendly for self } \\
\hline Anxious behavior, parent-report & 0.06 & -0.03 & -0.30 & 0.00 & 0.03 & $-0.36^{*}$ & -0.21 & -0.18 & -0.13 & -0.12 & -0.14 \\
\hline Present in common rooms, parent-report & 0.14 & 0.17 & 0.23 & 0.28 & 0.18 & $0.47 *$ & 0.30 & 0.25 & 0.27 & 0.23 & 0.10 \\
\hline \multicolumn{12}{|l|}{ Linda } \\
\hline & \multicolumn{11}{|c|}{ Mindfulness practice time } \\
\hline Reacting irritated to sounds & 0.09 & 0.28 & -0.10 & 0.15 & 0.11 & -0.02 & -0.09 & -0.01 & 0.00 & 0.09 & 0.21 \\
\hline
\end{tabular}

$* p<0.0045$

final week of the MYmind program. They had expected much more intense worry in relation to going to the new school. They explained that the worsening of Steven's goals in the intervention phase was mainly related to changes in his school situation. Steven's parent explained that Steven applied mindfulness practices to cope with his worries and mentioned that Steven asked less to stay home from school and his school attendance was higher than the year before. Steven reported that he continued practicing mindfulness
2 months and 1 year after the MYmind program had ended, which indicated that he benefitted from mindfulness. Also, his goals had improved again 1 year after the training, which may indicate that the mindfulness practice was associated with positive movement in terms of his goals in the long term. Together, the information suggests that though not reflected in the personal goals until much later, the mindfulness practice may have been beneficial for Steven, initially in order to cope with the stressors of going to a new school 
Fig. 3 Cross-lagged correlation results between David's presentmoment attention and parentreported presence in the common rooms during the 2-month follow-up. This indicated that the increase in parent-reported presence in the common rooms was both preceded and followed by the increase in presentmoment attention

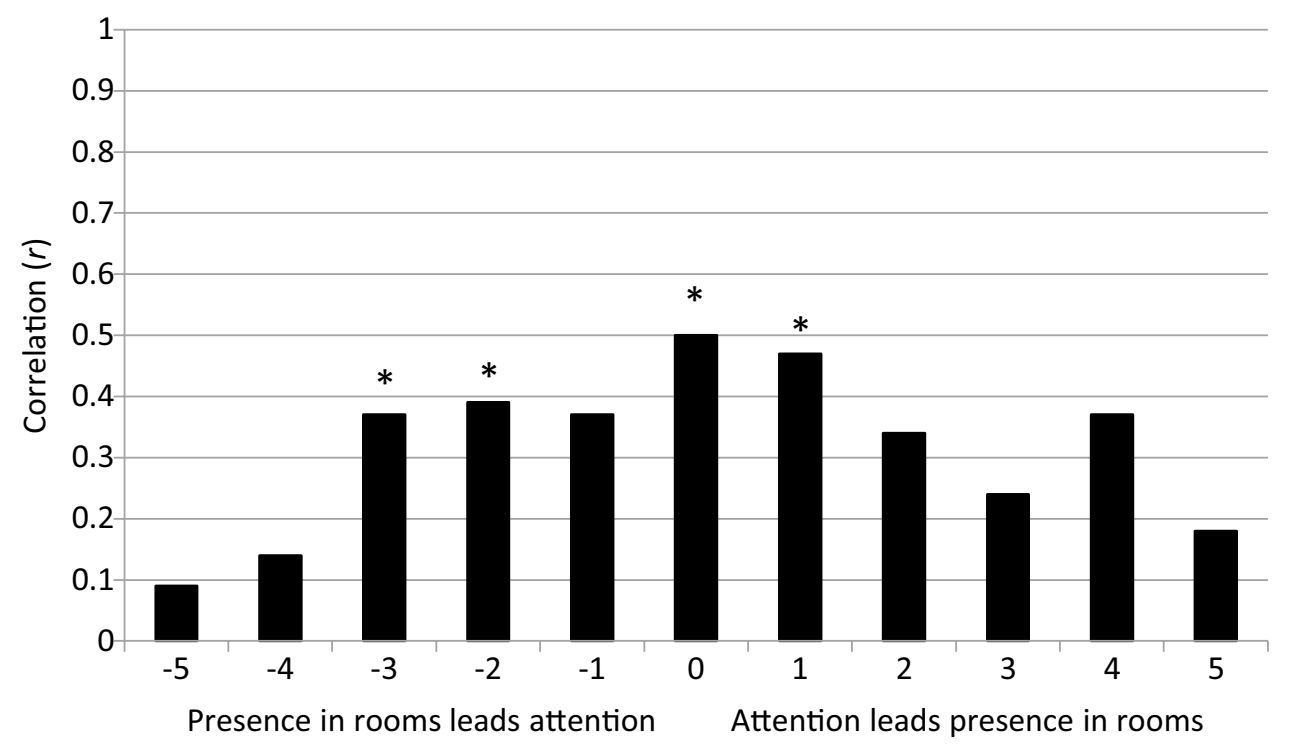

and later also in positive change on his goals. Stevens' case points toward methodological challenges where the personal clinical relevance of the intervention, in this case an MBP, may still be difficult to capture, even when using individualized items monitored on a day-to-day basis. Future case studies should systematically assess external coinciding life factors that could cause or explain adverse effects, as well as the benefits of MBPs as experienced by the participants as a way to cope with clinical symptoms that continue to be present.

The format of the MYmind program in the present study was different from the format of previous studies on MYmind. It consisted of individual sessions rather than group sessions, and one session for the parent rather than a full 9-week program of mindful parenting. The individual sessions lowered the threshold for the adolescents to speak about their experiences or having to interact with peers. Adolescents with autism and social anxiety disorder may particularly find it difficult to discuss their internal experiences during inquiry in a group format. The format also allowed more attention to the experiences of each adolescent, and the mindfulness trainers were positive about having more time to engage with their clients and apply the session content more directly to the individual and the individual's goals during the individual sessions compared to the group format. However, adolescents with autism that took part in a group MYmind program expressed that connecting with peers contributed to the experienced benefits (Ridderinkhof et al., 2019).

Regarding the parallel mindful parenting sessions, previous studies have shown that parallel mindful parenting sessions could decrease the heightened parental stress and mental health problems of parents (e.g., Ridderinkhof et al., 2018) and stand-alone mindful parenting programs have shown beneficial effects for children of participating parents (Bögels et al., 2014; Hwang et al., 2015; Meppelink et al., 2016; Neece, 2014). This format however makes the adolescents dependent on their parents' participation in an MBP. In the present study, a format with only one session for parents was used. The study suggests that this format could be a solution for adolescents with autism who are motivated for an MBP while their parents are not, or for adolescents who do not want their parents to be involved in their treatment. However, it is important for future studies to compare the different formats of MBPs for children and adolescents with autism focusing on the relative effectiveness and costeffectiveness and ideally identifying the effective elements of the protocol.

The secondary objective of the present study was to add knowledge on the change processes involved in the MYmind program. Interestingly, mindfulness practice time did not consistently precede positive change in the adolescents' personal goals. In the literature, the results on the role of home practice in MBPs are also inconclusive. A systematic review found a positive relation between home practice and clinical outcomes in only four out of seven studies (Lloyd et al., 2018) and a meta-analysis of 28 studies found a small positive relation between home practice and outcomes (Parsons et al., 2017). Thus, the results on the mediating role of mindfulness practice in MBPs are inconclusive, both in the present study and in previous literature.

Worry came forward as a possible mechanism of change, because decreased worry preceded improvements in withdrawn and anxious behavior for two adolescents. The MYmind program might have taught the adolescents to view thoughts as passing events in a wider field of awareness, so to decenter from their thoughts (Farb et al., 2018), which could stop repetitive negative thinking patterns. Several 
meta-analyses indicate that worry is a mechanism of change of MBPs for adults by which psychological functioning improves (Alsubaie et al., 2017; Gu et al., 2015). By worrying less about possible negative consequences, adolescents may have been better capable and felt more confident to engage in social interactions, with reductions in withdrawn and anxious behavior.

We expected stress reduction to be an important goal for adolescents with autism, and a potential mechanism of change for internalizing symptoms. However, none of the adolescents formulated decreasing stress as a primary personal goal. Further, stress only decreased during the intervention for one adolescent and in this case only preceded an improvement on one personal item. These results are conflicting with decrease in stress previously found for adolescents with autism after taking part in the MYmind program (Ridderinkhof et al., 2018), and the decreased psychological and physiological stress after MBPs that is found in other populations (e.g., Chiesa and Serretti, 2009; Morton et al., 2020; Pascoe et al., 2017). A possible explanation is that the adolescents did not phrase their stress responses as "stress" while the formulations of feeling anxious, feeling worried, and feeling irritated in the personal goals could reflect their stress responses. Future studies could combine psychological and physiological stress outcomes to shed light on the effects of an MBP on stress of adolescents with autism.

\section{Limitations and Future Research Directions}

The person-centered approach contained both strengths and limitations. First, the use of individualized items allowed each adolescent to complete questions that were relevant and understandable for them. The strength of this approach is that it addresses the difficulties often experienced by adolescents with autism when completing standardized tests which are developed for the general population. These difficulties may be linked to the autism symptom of interpreting language literally (APA, 2013). The individualized items further allowed a focus on what each adolescent found to be the most essential goal for participating in an MBP. A limitation of using individualized items is that it also restricts the generalizability across the five cases. Further, it may still not capture the clinical relevance of the MBP for the adolescent relative to contextual changes, which was likely the case for Steven. In addition, adolescent- and parent-report on personal goals did not correspond highly and showed variation in the results. This might reflect different interpretations of the item or the behavior. Second, monitoring and analyzing the outcomes for each individual over time form a strength as we could draw conclusions on the individual level, and thus provide evidence for within-person relationships on the potential mechanisms of change (Hamaker, 2012). The variation and mechanisms of change in the present study seem difficult to address in a group design. In group designs, information is aggregated across the group, which is strikingly often not generalizable to the individual level (Fisher et al., 2018). However, adolescents and parents experienced difficulties in completing the daily questionnaire in a daily fashion, resulting in missing values. The replacement of the missing values might have influenced the results of the analyses. Future studies could further improve the completion of daily questionnaires by using tailored modalities (e.g., text check-ins) and rewards. Moreover, because each case was conducted with a different mindfulness trainer, in a different center, and mostly a different country, we cannot exclude that these contextual factors influenced the results. Finally, combining visual inspection with statistical methods for single-case studies is seen as strengthening the systematic interpretation of the results.

Overall, the findings indicated that most of the adolescents with autism and associated internalizing conditions benefitted partially from the MYmind program. However, some of their internalizing symptoms did not improve, and one adolescent seemed to deteriorate. This study provides a detailed example of how individual cases within the clinical practice can be studied and documented to share and enhance knowledge and experiences on the struggles and benefits of providing and studying MBPs for adolescents with autism.

Author Contribution AR collaborated with designing the study, executed the study, analyzed the data, and wrote the first draft of the paper as part of her doctoral dissertation defended and approved by the University of Amsterdam in 2019. ME conceptualized the study, collaborated with the design and execution of the study at one site, and wrote the final manuscript. AR and ME shared first authorship. EIdB conceptualized the study, designed the study, supervised the execution of the study and the analysis of the data, and collaborated in the writing and editing of the manuscript. RB was part of the data collection and collaborated in the writing and editing of the manuscript. SSG was part of the data collection and collaborated in the writing and editing of the manuscript. JAW conceptualized the study, collaborated with the design and execution of the study at one site, and collaborated in the writing and editing of the manuscript. PvdM was part of the data collection and collaborated in the writing and editing of the manuscript. NNS collaborated with the design of the study and the analysis of the data and collaborated in the writing and editing of the manuscript. SMB collaborated with the design of the study and in the writing and editing of the final manuscript. Anna Ridderinkhof and Mette Elmose shared first authorship.

\section{Declarations}

Ethics Approval The study was performed in accordance with the ethical standards as laid down in the 1964 Declaration of Helsinki and its later amendments or comparable ethical standards. Ethical approval was received for each country. In the Netherlands, the Medical Ethics Committee of the Amsterdam Medical Center approved the study. In Canada, the Human Participants Ethics Review Sub-Committee of York University approved the study. In Denmark, the Regional Com- 
mittees on Health Research Ethics for Southern Denmark approved the study.

Informed Consent Written informed consent was obtained from all participants.

Conflict of Interest Author Susan M. Bögels owns shares in UvA minds, one of the participating treatment centers. The Canadian part of the study received support from Kids Brain Health Foundation. The other authors declare that they have no conflict of interest.

Open Access This article is licensed under a Creative Commons Attribution 4.0 International License, which permits use, sharing, adaptation, distribution and reproduction in any medium or format, as long as you give appropriate credit to the original author(s) and the source, provide a link to the Creative Commons licence, and indicate if changes were made. The images or other third party material in this article are included in the article's Creative Commons licence, unless indicated otherwise in a credit line to the material. If material is not included in the article's Creative Commons licence and your intended use is not permitted by statutory regulation or exceeds the permitted use, you will need to obtain permission directly from the copyright holder. To view a copy of this licence, visit http://creativecommons.org/licenses/by/4.0/.

\section{References}

Achenbach, T. M., \& Rescorla, L. A. (2001). Manual for the ASEBA School-Age Forms \& Profiles. University of Vermont, Research Centre for Children, Youth \& Families.

Alsubaie, M., Abbott, R., Dunn, B., Dickens, C., Keil, T. F., Henley, W., \& Kuyken, W. (2017). Mechanisms of action in mindfulnessbased cognitive therapy (MBCT) and mindfulness-based stress reduction (MBSR) in people with physical and/or psychological conditions: A systematic review. Clinical Psychology Review, 55, 74-91. https://doi.org/10.1016/j.cpr.2017.04.008

American Psychiatric Association. (2013). Diagnostic and statistical manual of mental disorders (5th ed.). American Psychiatric Association

Barlow, D. H., Nock, M., \& Hersen, M. (2009). Single-case experimental designs (3rd ed.). Allyn \& Bacon.

Bishop, S. R., Lau, M., Shapiro, S., Carlson, L., Anderson, N. D., Carmody, J., Segal, Z. V., Abbey, S., Speca, M., Velting, D., \& Devins, G. (2004). Mindfulness: A proposed operational definition. Clinical Psychology: Science and Practice, 11(3), 230-241. https://doi.org/10.1093/clipsy/bph077

Block-Lerner, J., Adair, C., Plumb, J. C., Rhatigan, D. L., \& Orsillo, S. M. (2007). The case for mindfulness-based approaches in the cultivation of empathy: Does nonjudgmental, present-moment awareness increase capacity for perspective-taking and empathic concern? Journal of Marital and Family Therapy, 33(4), 501-516. https://doi.org/10.1111/j.1752-0606.2007.00034

Bögels, S. M., Hellemans, J., van Deursen, S., Römer, M., \& van der Meulen, R. (2014). Mindful parenting in mental health care: Effects on parental and child psychopathology, parental stress, parenting, coparenting, and marital functioning. Mindfulness, 5(5), 536-551.

Borckardt, J. J., Nash, M. R., Murphy, M. D., Moore, M., Shaw, D., \& O'Neil, P. (2008). Clinical practice as natural laboratory for psychotherapy research: A guide to case-based time-series analysis. American Psychologist, 63(2), 77-95. https://doi.org/10.1037/ 0003-066X.63.2.77
Braet, C., Vandevivere, E., \& Wante, L. (2016). SCID-Junior. Unpublished manuscript of Ghent University.

Browning, J., Osborne, L. A., \& Reed, P. (2009). Research section: A qualitative comparison of perceived stress and coping in adolescents with and without autistic spectrum disorders as they approach leaving school. British Journal of Special Education, 36(1), 36-43. https://doi.org/10.1111/j.1467-8578.2008.00400.x

Cabin, R. J., \& Mitchell, R. J. (2000). To Bonferroni or not to Bonferroni: When and how are the questions. Bulletin of the Ecological Society of America, 81(3), 246-248.

Cardillo, J. E., \& Choate, R. O. (1994). Illustrations of goal setting. In T. Kiresuk, A. Smith, \& J. Cardillo (Eds.), Goal attainment scaling: Applications, theory, and measurement (pp. 15-37). Lawrence Erlbaum Associates.

Chiang, H. M., \& Wineman, I. (2014). Factors associated with quality of life in individuals with autism spectrum disorders: A review of literature. Research in Autism Spectrum Disorders, 8(8), 974-986. https://doi.org/10.1016/j.rasd.2014.05.003

Chiesa, A., \& Serretti, A. (2009). Mindfulness-based stress reduction for stress management in healthy people: A review and meta-analysis. The Journal of Alternative and Complementary Medicine, 15(5), 593-600. https://doi.org/10.1089/acm.2008.0495

Cohen, J. (1992). A power primer. Psychological Bulletin, 112(1), $155-159$.

Corbett, B. A., Schupp, C. W., Simon, D., Ryan, N., \& Mendoza, S. (2010). Elevated cortisol during play is associated with age and social engagement in children with autism. Molecular Autism, 1(1), 1-13. https://doi.org/10.1186/2040-2392-1-13

Coronado-Montoya, S., Levis, A. W., Kwakkenbos, L., Steele, R. J., Turner, E. H., \& Thombs, B. D. (2016). Reporting of positive results in randomized controlled trials of mindfulness-based mental health interventions. PLoS ONE, 11(4), 1-18. https://doi.org/ 10.1371/journal.pone. 0153220

De Bruin, E. I., Blom, R., Smit, F. M., van Steensel, F. J., \& Bögels, S. M. (2015). MYmind: Mindfulness training for youngsters with autism spectrum disorders and their parents. Autism, 19(8), 906914. https://doi.org/10.1177/1362361314553279

Dempster, A. P., Laird, N. M., \& Rubin, D. B. (1977). Maximum likelihood from incomplete data via the EM algorithm. Journal of the Royal Statistical Society, Series B, 39(1), 1-38.

Drotar, D. (2009). Editorial: Case studies and series: A call for action and invitation for submissions. Journal of Pediatric Psychology, 34(8), 795-802. https://doi.org/10.1093/jpepsy/jsp059

Dunning, D. L., Griffiths, K., Kuyken, W., Crane, C., Foulkes, L., Parker, J., \& Dalgleish, T. (2019). The effects of mindfulnessbased interventions on cognition and mental health in children and adolescents - a meta-analysis of randomized controlled trials. Journal of Child Psychology and Psychiatry and Allied Disciplines, 60(3), 244-258. https://doi.org/10.1111/jcpp.12980

Farb, N., Anderson, A., Ravindran, A., Hawley, L., Irving, J., Mancuso, E., Gulamani, T., Williams, G., Ferguson, A., \& Segal, Z. V. (2018). Prevention of relapse/recurrence in major depressive disorder with either mindfulness-based cognitive therapy or cognitive therapy. Journal of Consulting and Clinical Psychology, 86(2), 200-204. https://doi.org/10.1037/ccp0000266

Fisher, A. J., Medaglia, J. D., \& Jeronimus, B. F. (2018). Lack of group-to-individual generalizability is a threat to human subjects research. Proceedings of the National Academy of Sciences, 115(27), 6106-6115. https://doi.org/10.1073/pnas.1711978115

Flyvbjerg, B. (2006). Five misunderstandings about case-study research. Qualitative Inquiry, 12, 219-245. https://doi.org/10. 1177/1077800405284363

Ghaziuddin, M., Ghaziuddin, N., \& Greden, J. (2002). Depression in persons with autism: Implications for research and clinical care. Journal of Autism and Developmental Disorders, 32(4), 299-306. https://doi.org/10.1023/A:1016330802348 
Goldberg, S. B., Tucker, R. P., Greene, P. A., Davidson, R. J., Wampold, B. E., Kearney, D. J., \& Simpson, T. L. (2018). Mindfulnessbased interventions for psychiatric disorders: A systematic review and meta-analysis. Clinical Psychology Review, 59, 52-60. https:// doi.org/10.1016/j.cpr.2017.10.011

Gu, J., Strauss, C., Bond, R., \& Cavanagh, K. (2015). How do mindfulness-based cognitive therapy and mindfulness-based stress reduction improve mental health and wellbeing? A systematic review and meta-analysis of mediation studies. Clinical Psychology Review, 37, 1-12. https://doi.org/10.1016/j.cpr.2015.01.006

Guerry, J. D., \& Hastings, P. D. (2011). In search of HPA axis dysregulation in child and adolescent depression. Clinical Child and Family Psychology Review, 14(2), 135-160. https://doi.org/10. 1007/s10567-011-0084-5

Hamaker, E. L. (2012). Why researchers should think "within-person": A paradigmatic rationale. In M. R. Mehl \& T. A. Conner (Eds.), Handbook of research methods for studying daily life (pp. 43-61). Guilford Press.

Harnett, P. H., \& Dawe, S. (2012). Review: The contribution of mindfulness-based therapies for children and families and proposed conceptual integration. Child and Adolescent Mental Health, 17(4), 195-208. https://doi.org/10.1111/j.1475-3588.2011. 00643.X

Hwang, Y. S., Kearney, P., Klieve, H., Lang, W., \& Roberts, J. (2015). Cultivating mind: Mindfulness interventions for children with autism spectrum disorder and problem behaviours, and their mothers. Journal of Child and Family Studies, 24(10), 3093-3106. https://doi.org/10.1007/s10826-015-0114-x

Kabat-Zinn, J. (1982). An outpatient program in behavioral medicine for chronic pain patients based on the practice of mindfulness meditation: Theoretical considerations and preliminary results. General Hospital Psychiatry, 4(1), 33-47. https://doi.org/10.1016/ 0163-8343(82)90026-3

Kiep, M., Spek, A. A., \& Hoeben, L. (2015). Mindfulness-based therapy in adults with an autism spectrum disorder: Do treatment effects last? Mindfulness, 6(3), 637-644. https://doi.org/10.1007/ s12671-014-0299-x

Kuhlman, K. R., Olson, S. L., \& Lopez-Duran, N. L. (2014). Predicting developmental changes in internalizing symptoms: Examining the interplay between parenting and neuroendocrine stress reactivity. Developmental Psychobiology, 56(5), 908-923. https://doi.org/ 10.1002/dev.21166

Kuyken, W., Hayes, R., Barrett, B., Byng, R., Dalgleish, T., Kessler, D., Lewis, G., Watkins, E., Brejcha, C., Cardy, J., Causley, A., Cowderoy, S., Evans, A., Gradinger, F., Kaur, S., Lanham, P., Morant, N., Richards, J., Shah, P., ... \& Causley, A. (2015). Effectiveness and cost-effectiveness of mindfulness-based cognitive therapy compared with maintenance antidepressant treatment in the prevention of depressive relapse or recurrence (PREVENT): A randomized controlled trial. The Lancet, 386(9988), 63-73. https://doi.org/10.1016/S0140-6736(14)62222-4

Kuyken, W., Watkins, E., Holden, E., White, K., Taylor, R. S., Byford, S., Evans, A., Radford, S., Teasdale, J. D., \& Dalgleish, T. (2010). How does mindfulness-based cognitive therapy work? Behaviour Research and Therapy, 48(11), 1105-1112. https://doi.org/10. 1016/j.brat.2010.08.003

Lane, J. D., \& Gast, D. L. (2014). Visual analysis in single case experimental design studies: Brief review and guidelines. Neuropsychological Rehabilitation, 24(3-4), 445-463. https://doi.org/10.1080/ 09602011.2013 .815636

Leyfer, O. T., Folstein, S. E., Bacalman, S., Davis, N. O., Dinh, E., Morgan, J., Tager-Flusberg, H., \& Lainhart, J. E. (2006). Comorbid psychiatric disorders in children with autism: Interview development and rates of disorders. Journal of Autism and Developmental Disorders, 36(7), 849-861. https://doi.org/10.1007/ s10803-006-0123-0
Lindahl, J. R., Fisher, N. E., Cooper, D. J., Rosen, R. K., \& Britton, W. B. (2017). The varieties of contemplative experience: A mixedmethods study of meditation-related challenges in Western Buddhists. PLoS ONE, 12(5), 1-38. https://doi.org/10.1371/journal. pone. 0176239

Little, R. J. A. (1988). A test of missing completely at random for multivariate data with missing values. Journal of the American Statistical Association, 83(404), 1198-1202.

Lloyd, A., White, R., Eames, C., \& Crane, R. (2018). The utility of home-practice in mindfulness-based group interventions: A systematic review. Mindfulness, 9(3), 673-692. https://doi.org/10. 1007/s12671-017-0813-z

Lord, C., Risi, S., Lambrecht, L., Cook, E. H., Leventhal, B. L., DiLavore, P. C., Pickles, A., \& Rutter, M. (2000). The Autism Diagnostic Observation Schedule-Generic: A standard measure of social and communication deficits associated with the spectrum of autism. Journal of Autism and Developmental Disorders, 30(3), 205-223. https://doi.org/10.1023/A:1005592401947

Lord, C., Rutter, M., DiLavore, P. C., Risi, S., Gotham, K., \& Bishop, S. (2012). Autism diagnostic observation schedule (2nd ed.). Western Psychological Services.

Meppelink, R., de Bruin, E. I., Wanders-Mulder, F. H., Vennik, C. J., \& Bögels, S. M. (2016). Mindful parenting training in child psychiatric settings: Heightened parental mindfulness reduces parents' and children's psychopathology. Mindfulness, 7(3), 680-689. https:// doi.org/10.1007/s12671-016-0504-1

Morton, M. L., Helminen, E. C., \& Felver, J. C. (2020). A systematic review of mindfulness interventions on psychophysiological responses to acute stress. Mindfulness, 11, 2039-2054. https://doi. org/10.1007/s12671-020-01386-7

Neece, C. L. (2014). Mindfulness-based stress reduction for parents of young children with developmental delays: Implications for parental mental health and child behavior problems. Journal of Applied Research in Intellectual Disabilities, 27(2), 174-186. https://doi. org/10.1111/jar.12064

Newschaffer, C. J., Croen, L. A., Daniels, J., Giarelli, E., Grether, J. K., Levy, S. E., Mandell, D. S., Miller, L. A., Pinto-Martin, J., Reaven, J., Reynolds, A. M., Rice, C. E., Schendel, D., \& Windham, G. C. (2007). The epidemiology of autism spectrum disorders. Annual Review of Public Health, 28(1), 235-258. https://doi. org/10.1146/annurev.publhealth.28.021406.144007

Parsons, C. E., Crane, C., Parsons, L. J., Fjorback, L. O., \& Kuyken, W. (2017). Home practice in mindfulness-based cognitive therapy and mindfulness-based stress reduction: A systematic review and meta-analysis of participants' mindfulness practice and its association with outcomes. Behaviour Research and Therapy, 95, 29-41. https://doi.org/10.1016/j.brat.2017.05.004

Pascoe, M. C., Thompson, D. R., Jenkins, Z. M., \& Ski, C. F. (2017). Mindfulness mediates the physiological markers of stress: Systematic review and meta-analysis. Journal of Psychiatric Research, 95, 156-178. https://doi.org/10.1016/j.jpsychires.2017.08.004

Ridderinkhof, A., de Bruin, E. I., Blom, R., \& Bögels, S. M. (2018). Mindfulness-based program for children with autism spectrum disorder and their parents: Direct and long-term improvements. Mindfulness, 9(3), 773-791. https://doi.org/10.1007/ s12671-017-0815-x

Ridderinkhof, A., de Bruin, E. I., Blom, R., Singh, N. N., \& Bögels, S. M. (2019). Mindfulness-based program for autism spectrum disorder: A qualitative study of the experiences of children and parents. Mindfulness, 10(9), 1936-1951. https://doi.org/10.1007/ s12671-019-01202-x

Rozental, A., Castonguay, L., Dimidjian, S., Lambert, M., Shafran, R., Andersson, G., \& Carlbring, P. (2018). Negative effects in psychotherapy: Commentary and recommendations for future research and clinical practice. Bjpsych Open, 4(4), 307-312. https://doi. org/10.1192/bjo.2018.42 
Salem-Guirgis, S., Albaum, C., Tablon, P., Riosa, P. B., Nicholas, D. B., Drmic, I. E., \& Weiss, J. A. (2019). MYmind: A concurrent group-based mindfulness intervention for youth with autism and their parents. Mindfulness, 10(9), 1730-1743. https://doi.org/10. 1007/s12671-019-01107-9

Schall, C. M., \& McDonough, J. T. (2010). Autism spectrum disorders in adolescence and early adulthood: Characteristics and issues. Journal of Vocational Rehabilitation, 2010(2), 81-88. https://doi. org/10.3233/JVR-2010-0503

Segal, Z. V., Williams, J. M. G., \& Teasdale, J. D. (2012). Mindfulnessbased cognitive therapy for depression. Guilford Press.

Silverman, W. K., \& Albano, A. M. (1996). The anxiety disorders interview schedule for children for DSM-IV $(A D I S-C / P)$. Psychological Corporation.

Simonoff, E., Pickles, A., Charman, T., Chandler, S., Loucas, T., \& Baird, G. (2008). Psychiatric disorders in children with autism spectrum disorders: Prevalence, comorbidity, and associated factors in a population-derived sample. Journal of the American Academy of Child \& Adolescent Psychiatry, 47(8), 921-929. https://doi.org/10.1097/CHI.0b013e318179964f

Singh, N. N., Lancioni, G. E., Manikam, R., Winton, A. S., Singh, A. N., Singh, J., \& Singh, A. D. (2011a). A mindfulness-based strategy for self-management of aggressive behavior in adolescents with autism. Research in Autism Spectrum Disorders, 5(3), 1153-1158. https://doi.org/10.1016/j.rasd.2010.12.012

Singh, N. N., Lancioni, G. E., Singh, A. D., Winton, A. S., Singh, A. N., \& Singh, J. (2011b). Adolescents with Asperger syndrome can use a mindfulness-based strategy to control their aggressive behavior. Research in Autism Spectrum Disorders, 5(3), 11031109. https://doi.org/10.1016/j.rasd.2010.12.006

Smith, J. D., Borckardt, J. J., \& Nash, M. R. (2012). Inferential precision in single-case time-series data streams: How well does the EM procedure perform when missing observations occur in autocorrelated data? Behavior Therapy, 43(3), 679-685. https://doi. org/10.1016/j.beth.2011.10.001

Spek, A. A., van Ham, N. C., \& Nyklíček, I. (2013). Mindfulness-based therapy in adults with an autism spectrum disorder: A randomized controlled trial. Research in Developmental Disabilities, 34(1), 246-253. https://doi.org/10.1016/j.ridd.2012.08.009

Spratt, E. G., Nicholas, J. S., Brady, K. T., Carpenter, L. A., Hatcher, C. R., Meekins, K. A., Furlanetto, R. W., \& Charles, J. M. (2012).
Enhanced cortisol response to stress in children in autism. Journal of Autism and Developmental Disorders, 42(1), 75-81. https://doi. org/10.1007/s10803-011-1214-0

Van Dam, N. T., van Vugt, M. K., Vago, D. R., Schmalzl, L., Saron, C. D., Olendzki, A., Meissner, T., Lazar, S. W., Kerr, C. E., Gorchov, J., Fox, K. C. R., Field, B. A., Britton, W. B., Brefczynski-Lewis, J. A., \& Meyer, D. E. (2018). Mind the hype: A critical evaluation and prescriptive agenda for research on mindfulness and meditation. Perspectives on Psychological Science, 13(1), 36-61. https:// doi.org/10.1177/1745691617709589

Van Steensel, F. J., Bögels, S. M., \& De Bruin, E. I. (2013a). Psychiatric comorbidity in children with autism spectrum disorders: A comparison with children with ADHD. Journal of Child and Family Studies, 22(3), 368-376. https://doi.org/10.1007/ s10826-012-9587-z

Van Steensel, F. J., Bögels, S. M., \& Perrin, S. (2011). Anxiety disorders in children and adolescents with autistic spectrum disorders: A meta-analysis. Clinical Child and Family Psychology Review, 14(3), 302-317. https://doi.org/10.1007/s10567-011-0097-0

Van Steensel, F. J., Dirksen, C. D., \& Bögels, S. M. (2013b). A cost of illness study of children with high-functioning autism spectrum disorders and comorbid anxiety disorders as compared to clinically anxious and typically developing children. Journal of Autism and Developmental Disorders, 43(12), 2878-2890. https://doi.org/ 10.1007/s10803-013-1835-6

Wechsler, D. (2011). Wechsler abbreviated scale of intelligence, second edition (WASI-II). NCS Pearson.

Winbush, N. Y., Gross, C. R., \& Kreitzer, M. J. (2007). The effects of mindfulness-based stress reduction on sleep disturbance: A systematic review. EXPLORE: The Journal of Science and Healing, 3(6), 585-591. https://doi.org/10.1016/j.explore.2007.08.003

Wong, S. Y., Chan, J. Y., Zhang, D., Lee, E. K., \& Tsoi, K. K. (2018). The safety of mindfulness-based interventions: A systematic review of randomized controlled trials. Mindfulness, 9(5), 1344 1357. https://doi.org/10.1007/s12671-018-0897-0

Publisher's Note Springer Nature remains neutral with regard to jurisdictional claims in published maps and institutional affiliations. 